\title{
Klein four-group and Darboux duality in conformal mechanics
}

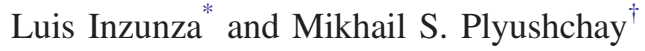 \\ Departamento de Física, Universidad de Santiago de Chile, Casilla 307, Santiago, Chile
}

(Received 25 April 2019; published 27 June 2019)

\begin{abstract}
We study the Klein four-group $\left(K_{4}\right)$ symmetry of the time-dependent Schrödinger equation for the conformal mechanics model of de Alfaro-Fubini-Furlan (AFF) with confining harmonic potential and coupling constant $g=\nu(\nu+1) \geq-1 / 4$. We show that it undergoes a complete or partial (at half-integer $\nu$ ) breaking on eigenstates of the system, and is the automorphism of the $\mathfrak{o} \mathfrak{g} \mathfrak{p}(2,2)$ superconformal symmetry in super-extensions of the model by inducing a transformation between the exact and spontaneously broken phases of $\mathcal{N}=2$ Poincaré supersymmetry. We exploit the $K_{4}$ symmetry and its relation with the conformal symmetry to construct the dual Darboux transformations which generate spectrally shifted pairs of the rationally deformed AFF models. Two distinct pairs of intertwining operators originated from Darboux duality allow us to construct complete sets of the spectrum generating ladder operators that identify specific finite-gap structure of a deformed system and generate three distinct related versions of nonlinearly deformed $\mathfrak{g l}(2, \mathbb{R})$ algebra as its symmetry. We show that at half-integer $\nu$, the Jordan states associated with confluent Darboux transformations enter the construction, and the spectrum of rationally deformed AFF systems undergoes structural changes.
\end{abstract}

DOI: $10.1103 /$ PhysRevD.99.125016

\section{INTRODUCTION}

In quantum mechanics, symmetries map the states of a system into its states. If the ground state is invariant under the corresponding transformations, one says that the symmetry is unbroken, otherwise symmetry is (spontaneously) broken. Sometimes, along with a continuous group of symmetry transformations, a discrete symmetry group appears [1], and a nontrivial interplay may occur between both types of symmetries. An interesting and important case from this point of view is presented by the conformal mechanics model of de Alfaro, Fubini and Furlan (AFF) [2] with confining harmonic potential and coupling constant $g=$ $\nu(\nu+1) \geq-1 / 4$. $^{1}$ Its nonrelativistic conformal symmetry and supersymmetric extensions [5-10] find a variety of interesting applications including the particles dynamics in black hole backgrounds [11-16], cosmology [17-19], nonrelativistic AdS/CFT correspondence [20-24], QCD

\footnotetext{
*luis.inzunza@usach.cl

†mikhail.plyushchay@usach.cl

${ }^{1}$ The AFF model is a two-particle Calogero system [3] with included confining harmonic potential term but omitted center of mass coordinate. Its Schrödinger operator also is known as the Gol'dman-Krivchenkov Hamiltonian [4].

Published by the American Physical Society under the terms of the Creative Commons Attribution 4.0 International license. Further distribution of this work must maintain attribution to the author(s) and the published article's title, journal citation, and DOI. Funded by SCOAP ${ }^{3}$.
}

confinement problem $[25,26]$, and physics of BoseEinstein condensates $[27,28]$.

On the other hand, the time-dependent Schrödinger equation for the AFF conformal mechanics model reveals a discrete Klein four-group symmetry generated by transformation of the parameter $\nu \rightarrow-\nu-1$, and by the spatial Wick rotation $x \rightarrow i x$ accompanied by the time reflection $t \rightarrow-t$. In the picture of the stationary Schrödinger equation the time reflection transforms into the change of the eigenvalue's sign $E \rightarrow-E$. The discrete symmetry $K_{4}$, however, turns out to be completely broken at the level of the quantum states when $\nu$ is not a half-integer number: application of the group generators to physical eigenstates produces formal eigenstates which do not satisfy the necessary boundary conditions. In the case of half-integer values of the parameter $\nu$ the $K_{4}$ discrete symmetry breaks partially, and transformation $\nu \rightarrow-\nu-1$, as we shall see, turns out to be a true symmetry nontrivially realized on the spectrum of the system. In the physics of anyons, where the AFF model is used to generate the transmutation of statistics, half-integer values of $\nu$ correspond to the twoparticle system of identical fermions [29-31]. In the context of the problem we consider here, even though the new solutions with arbitrary value of $\nu$ generated by transformations of the discrete group are not acceptable from the physical point of view, the analogs of such nonphysical states in other quantum systems are used to produce new solvable potentials and supersymmetric extensions via the (generalized) Darboux transformations [32-38]. They also are used, for example, for the construction of multisoliton 
and finite-gap solutions to the Korteweg-de Vries equation $[34,39,40]$. In particular, solutions with behavior typical for extreme waves are constructed in [41] based on nonphysical states of the AFF model without the harmonic trap.

The implications of the presence of the $K_{4}$ discrete symmetry group in the AFF model, and its interplay with conformal and superconformal symmetries, surprisingly, have not been investigated yet in the literature.

In this work, we study in detail the action of transformations of the Klein four-group on the states of the AFF system, its relation to the conformal symmetry, and its nontrivial role in $\mathcal{N}=2$ super-extensions of the AFF model and their $\mathfrak{o} \mathfrak{s} \mathfrak{p}(2,2)$ superconformal symmetry. This superconformal symmetry, as will be shown, is based essentially on the simplest case of the Darboux dual schemes which produce the same but spectrally shifted pairs of the quantum systems. Based on this observation, with the help of the Klein four-group transformations we address the problem of construction of the general Darboux dual schemes to generate infinite families of the new exactly solvable systems given by rational deformations of the conformal mechanics systems with arbitrary values of the parameter $\nu$. The general dual Darboux schemes that we obtain produce the same but spectrally shifted pairs of rationally deformed AFF models. As a consequence, their distinct intertwining operators allow us to construct the complete sets of the spectrum generating ladder operators for them and identify the nonlinearly deformed versions of conformal $\mathfrak{i l}(2, \mathbb{R})$ algebra which describe their symmetries. In this way we generalize our earlier results obtained for the restricted case of the AFF model with integer values of $\nu$ only [42,43], that were based on the Darboux transformations of the quantum harmonic oscillator. Coherently with the indicated above peculiarity of the half-integer values of the parameter $\nu$ from the point of view of the Klein four-group transformations, we will see how the Jordan states [41,44-49] enter the construction at $\nu=\mathbb{Z}+1 / 2$ via the confluent Darboux transformations. We also trace out the structural changes in the spectra of the rationally deformed AFF systems which happen at half-integer $\nu$ under continuous variation of this parameter.

The remainder of the paper is organized as follows. In Sec. II, we summarize the basic ingredients of the Darboux transformations, and in their context, consider the Jordan states. In Sec. III, we briefly present the AFF model together with its conformal symmetry and solutions of equations of motion provided by it, and discuss shortly the introduction of the scale which turns out to be related to the holographic QCD, the $\mathrm{AdS}_{2}$ isometry and the AdS/CFT correspondence, and Dirac's different forms of relativistic dynamics. Section IV is devoted to the discussion of the discrete Klein four-group symmetry of the time-dependent Schrödinger equation for the AFF model and its action on physical states, its relation to the conformal symmetry, and the role played by the discrete $K_{4}$ group in the $\mathcal{N}=2$ super-extensions of the AFF system generated by the simplest dual Darboux schemes. The algorithm of construction of the general dual Darboux schemes is developed in Sec. V. In Sec. VI we list some Darboux schemes which produce distinct infinite families of new exactly solvable systems described by rational deformations of the AFF model with arbitrary number of gaps introduced into the equidistant spectrum of the original system. For each such a Darboux scheme we construct its dual that plays a key role in identifying the spectral properties of the generated system and its symmetry. We also trace out there the structural changes in the spectra of the obtained systems under continuous variation of the parameter $\nu$. In Sec. VII, we use the intertwining operators of the dual Darboux schemes to construct the complete sets of the spectrum generating ladder operators of the rationally deformed AFF systems. These higher order differential operators detect and describe the "finite-gap" structure of the spectra of the corresponding systems, and generate three distinct but related versions of the nonlinearly deformed $\mathfrak{g} \mathfrak{l}(2, \mathbb{R})$ conformal algebra which describe their symmetries. The application of the general results of Secs. V-VII is illustrated by an example presented in Sec. VIII. In Sec. IX we summarize the results and discuss some problems to be interesting for further investigation. In three Appendixes some technical details necessary for the main text are presented.

\section{GENERALIZED DARBOUX TRANSFORMATIONS}

In this section we summarize some properties of the generalized Darboux transformations which will be employed in what follows.

\section{A. Darboux transformations and intertwining operators}

Consider the equation

$$
L_{0} \psi_{\lambda}=\lambda \psi_{\lambda}, \quad L_{0}=-\frac{d^{2}}{d x^{2}}+V(x),
$$

corresponding to the eigenvalue problem of a Schrödinger type operator $L_{0}$. In this section we treat Eq. (2.1) as a formal second order differential equation on some interval $(a, b)$, and in the following sections we take care about its physical nature. Suppose we have a set of solutions $\psi_{k}$ corresponding to eigenvalues $\lambda_{k}, k=1, \ldots, n$. We use them as seed states for generalized Darboux transformation and generate the associated eigenvalue problem

$$
\begin{aligned}
L_{[n]} \Psi_{\lambda} & =\lambda \Psi_{\lambda}, \\
L_{[n]} & =-\frac{d^{2}}{d x^{2}}+V(x)-2 \frac{d^{2}}{d x^{2}} \ln W\left(\psi_{1}, \ldots, \psi_{n}\right) .
\end{aligned}
$$


If the set of the seed states is chosen in such a way that the Wronskian $W$ takes nonzero values on $(a, b)$, then potential of the generated system will also be nonsingular there. In the general case, solutions of (2.2) are generated from corresponding solutions of Eq. (2.1),

$$
\Psi_{\lambda}=\frac{W\left(\psi_{1}, \ldots, \psi_{n}, \psi_{\lambda}\right)}{W\left(\psi_{1}, \ldots, \psi_{n}\right)}=\mathbb{A}_{n} \psi_{\lambda},
$$

where $\mathbb{A}_{n}$ is the differential operator of order $n$ defined recursively as

$$
\begin{aligned}
\mathbb{A}_{n} & =A_{n} \ldots A_{1}, & A_{k} & =\mathbb{A}_{k-1} \psi_{k} \frac{d}{d x}\left(\frac{1}{\mathbb{A}_{k-1} \psi_{k}}\right), \\
k & =1, \ldots, n, \quad & \mathbb{A}_{0} & =1 .
\end{aligned}
$$

By the construction, $\operatorname{ker} \mathbb{A}_{n}=\operatorname{span}\left\{\psi_{1}, \ldots, \psi_{n}\right\}$. Operator $\mathbb{A}_{n}$ and its Hermitian conjugate $\mathbb{A}_{n}^{\dagger}$ intertwine the operators $L_{0}$ and $L_{[n]}$,

$$
\mathbb{A}_{n} L_{0}=L_{[n]} \mathbb{A}_{n}, \quad \mathbb{A}_{n}^{\dagger} L_{[n]}=L_{0} \mathbb{A}_{n}^{\dagger},
$$

and satisfy relations

$\mathbb{A}_{n}^{\dagger} \mathbb{A}_{n}=\prod_{k=1}^{n}\left(L_{0}-\lambda_{k}\right), \quad \mathbb{A}_{n} \mathbb{A}_{n}^{\dagger}=\prod_{k=1}^{n}\left(L_{[n]}-\lambda_{k}\right)$.

From the first equation in (2.6) one can find that $\operatorname{ker} \mathbb{A}_{n}^{\dagger}=\operatorname{span}\left\{\mathbb{A}_{n} \tilde{\psi}_{1}, \ldots, \mathbb{A}_{n} \tilde{\psi}_{n}\right\}$, where

$$
\tilde{\psi}_{\lambda}=\psi_{\lambda} \int^{x} \frac{d \zeta}{\left(\psi_{\lambda}(\zeta)\right)^{2}}
$$

is a linear independent solution of Eq. (2.1) with the same eigenvalue $\lambda, W\left(\psi_{\lambda}, \tilde{\psi}_{\lambda}\right)=1$. Similarly to (2.3), $\mathbb{A}_{n}^{\dagger} \Psi_{\lambda}=$ $\psi_{\lambda}$ for $\Psi_{\lambda} \notin \operatorname{ker} \mathbb{A}_{n}^{\dagger}$, and

$$
\mathbb{A}_{n}^{\dagger}\left(\widetilde{\mathbb{A}_{n} \tilde{\psi}_{k}}\right)=\psi_{k} \in \operatorname{ker} \mathbb{A}_{n} .
$$

Here and in what follows we consider equalities between wave functions and Wronskians in "up to a multiplicative constant" sense when the corresponding constant will be inessential.

The generalized Darboux transformation possesses the iterative property according to which system (2.2) can be generated alternatively via successive Darboux transformations. $^{2}$ This property allows us to get some useful Wronskian identities. Suppose that we have two collections of (formal) eigenstates of (2.1), $\left\{\phi_{n}\right\}=\left(\phi_{1}, \ldots, \phi_{n}\right)$ and $\left\{\varphi_{l}\right\}=\left(\varphi_{1}, \ldots, \varphi_{l}\right)$. In the first step, we generate a

\footnotetext{
${ }^{2}$ Intermediate systems appearing in such a way may have a singular nature on the interval $(a, b)[39,50,51]$.
}

Darboux transformation by taking the first collection as the set of the seed states, and obtain the intermediate Hamiltonian operator with potential $V_{1}=V(x)-$ $2\left(\ln W\left(\left\{\phi_{n}\right\}\right)\right)^{\prime \prime}$. In this way, the states of the second collection $\left\{\varphi_{l}\right\}$ will be mapped into the set of (formal in general case) eigenstates $\left\{\mathbb{A}_{n} \varphi_{l}\right\}=\left(\mathbb{A}_{n} \varphi_{1}, \ldots, \mathbb{A}_{n} \varphi_{l}\right)$ of the intermediate system, where $\mathbb{A}_{n}$ is the corresponding intertwining operator of order $n$ constructed following (2.4). Then, employing these states as the seed states for a second Darboux transformation, we finally obtain a Schrödinger operator with a potential $V_{2}=V_{1}(x)-$ $2\left(\ln W\left(\left\{\mathbb{A}_{n} \varphi_{l}\right\}\right)\right)^{\prime \prime}$. Having in mind that the same result will be produced by a one-step generalized Darboux transformation based on the whole set of the chosen eigenstates of the system $L_{0}$, we obtain the equality

$W\left(\left\{\phi_{n}\right\}\right) W\left(\left\{\mathbb{A}_{n} \varphi_{l}\right\}\right)=W\left(\phi_{1}, \ldots, \phi_{n}, \varphi_{1}, \ldots, \varphi_{l}\right)$.

Consider now the set of two states corresponding to a same eigenvalue $\lambda_{j},\left\{\phi_{2}\right\}=\left(\phi_{1}=\psi_{j}, \phi_{2}=\tilde{\psi}_{j}\right)$. In this case $W\left(\psi_{j}, \tilde{\psi}_{j}\right)=1$, and the corresponding intertwining operator reduces to $\mathbb{A}_{2}=-\left(L_{0}-\lambda_{j}\right)$. Using this observation and Eq. (2.8), we derive the equality $W\left(\psi_{j}, \tilde{\psi}_{j}\right.$, $\left.\varphi_{1}, \ldots, \varphi_{l}\right)=W\left(\left\{\varphi_{l}\right\}\right)$, which is generalized for the relation

$$
W\left(\psi_{1}, \tilde{\psi}_{1}, \ldots, \psi_{s}, \tilde{\psi}_{s}, \varphi_{1}, \ldots, \varphi_{l}\right)=W\left(\left\{\varphi_{l}\right\}\right) .
$$

In the case when functions $\varphi_{1}, \ldots, \varphi_{l}$ are not obligatorily to be eigenstates of the operator $L_{0}$, the last relation changes for

$$
\begin{array}{r}
W\left(\psi_{1}, \tilde{\psi}_{1}, \ldots, \psi_{s}, \tilde{\psi}_{s}, \varphi_{1}, \ldots, \varphi_{l}\right) \\
\quad=W\left(\left\{\prod_{k=1}^{s}\left(-L_{0}+\lambda_{k}\right) \varphi_{l}\right\}\right) .
\end{array}
$$

\section{B. Jordan states}

For a given Schrödinger operator $L_{0}$, one can construct a certain set of functions which are not its eigentstates but are annihilated by the action of a certain polynomial of $L_{0}$. Functions of such a nature can be related with the confluent Darboux transformations and are identified as Jordan states [45]. They were used, for example, in the construction of isospectral deformations of the harmonic oscillator systems $[48,52]$, and also they appeared in the context of solutions to the KdV equation [41]. In this subsection we construct Jordan states that are solutions of the fourth order differential equation $\left(L_{0}-\lambda_{*}\right)^{2} \chi_{*}=0$. They will play an important role in subsequent consideration.

We employ the following approach: take an eigenstate $\psi_{*}$ corresponding to eigenvalue $\lambda_{*}$ as a seed state of the Darboux transformation. This provides us with the first order differential operators 


$$
A_{\psi_{*}}=\psi_{*} \frac{d}{d x}\left(\frac{1}{\psi_{*}}\right), \quad A_{\psi_{*}}^{\dagger}=-\frac{1}{\psi_{*}} \frac{d}{d x} \psi_{*}
$$

According to Eq. (2.6), their product gives us the shifted Schrödinger operator $A_{\psi_{*}}^{\dagger} A_{\psi_{*}}=L_{0}-\lambda_{*}$, whose kernel is spanned by the linear independent states $\psi_{*}$ and $\tilde{\psi}_{*}$. The problem of constructing Jordan states reduces then to solving equations

$$
\begin{aligned}
& A_{\psi_{*}}^{\dagger} A_{\psi_{*}} \Omega_{*}=\left(L_{0}-\lambda_{*}\right) \Omega_{*}=\psi_{*}, \\
& A_{\psi_{*}}^{\dagger} A_{\psi_{*}} \breve{\Omega}_{*}=\left(L_{0}-\lambda_{*}\right) \breve{\Omega}_{*}=\tilde{\psi}_{*} .
\end{aligned}
$$

Their solutions are given, up to a linear combination of $\psi_{*}$ and $\tilde{\psi}_{*}$, by particular solutions of respective inhomogeneous equations,

$$
\begin{aligned}
& \Omega_{*}=\psi_{*} \int_{a}^{x} \frac{d \zeta}{\psi_{*}^{2}(\zeta)} \int_{\zeta}^{b} \psi_{*}^{2}(\eta) d \eta, \\
& \breve{\Omega}_{*}=\psi_{*} \int_{a}^{x} \frac{d \zeta}{\psi_{*}^{2}(\zeta)} \int_{\zeta}^{b} \psi_{*}(\eta) \tilde{\psi}_{*}(\eta) d \eta .
\end{aligned}
$$

Here the integration limits are chosen coherently with the region where the operator $L_{0}$ is defined, and we have the relations

$W\left(\psi_{*}, \Omega_{*}\right)=\int_{x}^{b} \psi_{*}^{2} d \zeta, \quad W\left(\psi_{*}, \breve{\Omega}_{*}\right)=\int_{x}^{b} \psi_{*} \tilde{\psi}_{*} d \zeta$,

which will be useful to produce nonsingular confluent Darboux transformations.

Let us inspect now the role of Jordan states (2.13) in Darboux transformations generated by a set of the seed states $\left\{\psi_{n}\right\}$. The intertwining operator (2.4) and Eqs. (2.5) and (2.12) give us the relations

$$
\mathbb{A}_{n} \psi_{*}=\left(L_{[n]}-\lambda_{*}\right) \mathbb{A}_{n} \Omega_{*}, \quad \mathbb{A}_{n} \tilde{\psi}_{*}=\left(L_{[n]}-\lambda_{*}\right) \mathbb{A}_{n} \breve{\Omega}_{*} .
$$

If the state $\psi_{*}\left(\right.$ or $\left.\tilde{\psi}_{*}\right)$ is annihilated by $\mathbb{A}_{n}$, i.e., if the set of the seed states $\left\{\psi_{n}\right\}$ includes $\psi_{*}\left(\right.$ or $\left.\tilde{\psi}_{*}\right)$, the function $\mathbb{A}_{n} \Omega_{*}$ (or $\mathbb{A}_{n} \breve{\Omega}_{*}$ ) will be an eigenstate of $L_{[n]}$ with eigenvalue $\lambda_{*}$ which is available to produce another Darboux transformation if we consider $L_{[n]}$ as an intermediate system. Otherwise, the indicated function is a Jordan state of $L_{[n]}$, and in correspondence with (2.13) we have

$$
\begin{aligned}
& \mathbb{A}_{n} \Omega_{*}=\left(\mathbb{A}_{n} \psi_{*}\right) \int_{a}^{x} \frac{d \zeta}{\left(\mathbb{A}_{n} \psi_{*}\right)^{2}(\zeta)} \int_{\zeta}^{b}\left(\mathbb{A}_{n} \psi_{*}\right)^{2}(\eta) d \eta \\
& \mathbb{A}_{n} \breve{\Omega}_{*}=\left(\mathbb{A}_{n} \psi_{*}\right) \int_{a}^{x} \frac{d \zeta}{\left(\mathbb{A}_{n} \psi_{*}\right)^{2}(\zeta)} \int_{\zeta}^{b}\left(\mathbb{A}_{n} \psi_{*}\right)(\eta) \widetilde{\mathbb{A}_{n} \psi_{*}}(\eta) d \eta
\end{aligned}
$$

up to a linear combination with $\mathbb{A}_{n} \psi_{*}$ and $\widetilde{\mathbb{A}_{n} \psi_{*}}$.

Having in mind that Jordan states appear naturally in the confluent generalized Darboux transformations [45], one can consider directly a generalized Darboux transformation based on the following set of the seed states: $\left(\psi_{1}, \Omega_{1}, \ldots\right.$, $\left.\psi_{n}, \Omega_{n}\right)$. This generates a Darboux-transformed system which we denote by $\hat{L}_{[2 n]}$. The intertwining operator $\mathbb{A}_{2 n}^{\Omega}$ as a differential operator of order $2 n$ is built according to the same rule (2.4), but with the inclusion of Jordan states into the set of generating functions. By the construction, this operator annihilates the chosen $2 n$ seed states, and one can show that

$$
\begin{aligned}
& \left(\mathbb{A}_{2 n}^{\Omega}\right)^{\dagger} \mathbb{A}_{2 n}^{\Omega}=\prod_{i=1}^{n}\left(L-\lambda_{i}\right)^{2}, \\
& \mathbb{A}_{2 n}^{\Omega}\left(\mathbb{A}_{2 n}^{\Omega}\right)^{\dagger}=\prod_{i}^{n}\left(\hat{L}_{[2 n]}-\lambda_{i}\right)^{2} .
\end{aligned}
$$

This, in particular, means that $\operatorname{ker}\left(\mathbb{A}_{2 n}^{\Omega}\right)^{\dagger}=\operatorname{span}\left\{\mathbb{A}_{2 n}^{\Omega} \tilde{\psi}_{1}\right.$, $\left.\mathbb{A}_{2 n}^{\Omega} \breve{\Omega}_{1}, \ldots, \mathbb{A}_{2 n}^{\Omega} \tilde{\psi}_{n}, \mathbb{A}_{2 n}^{\Omega} \breve{\Omega}_{n}\right\}$. In the context of generalized Darboux transformations based on a mixture of eigenstates and Jordan states, a useful relation

$$
W\left(\psi_{*}, \tilde{\psi}_{*}, \Omega_{*}, \breve{\Omega}_{*}, \varphi_{1}, \ldots, \varphi_{l}\right)=W\left(\varphi_{1}, \ldots, \varphi_{l}\right)
$$

can be obtained by employing Eq. (2.10) with $s=1$, and Eqs. (2.12) and (2.9), Here we imply that $\varphi_{i}$ with $i=$ $1, \ldots, l$ is the set of solutions of equation (2.1) with $\lambda_{i} \neq \lambda_{*}$.

\section{AFF CONFORMAL MECHANICS MODEL}

Consider now the one-dimensional system given by the action [2]

$$
I[q]=\int L(q, \dot{q}) d t, \quad L=\frac{1}{2}\left(\dot{q}^{2}-\frac{g}{q^{2}}\right),
$$

where $q>0$ has dimension $[q]=[\sqrt{t}]$, and $g$ is a dimensionless coupling constant which classically is assumed to be positive to avoid the "problem of fall to the center." System (3.1) is characterized by conformal symmetry $\operatorname{Conf}\left(\mathbb{R}^{1}\right)$ that canonically is generated by the Hamiltonian $H_{g}$, the dilatation generator $D$, and generator of special conformal transformations $K$,

$$
\begin{aligned}
H_{g} & =\frac{1}{2}\left(p^{2}+\frac{g}{q^{2}}\right), \\
D & =\frac{1}{4}(q p+p q)-H_{g} t, \\
K & =\frac{1}{2} q^{2}-2 D t-H_{g} t^{2},
\end{aligned}
$$


where $p=\dot{q}$. These are the integrals of motion that satisfy the equation of the form $\frac{d}{d t} A=\frac{\partial A}{\partial t}+\{A, H\}=0$. They obey the $\mathfrak{s} \mathfrak{o}(2,1)$ algebra $^{3}$

$$
\left\{D, H_{g}\right\}=H_{g}, \quad\{D, K\}=-K, \quad\left\{H_{g}, K\right\}=-2 D,
$$

which is isomorphic to the algebra $\mathfrak{g} \mathfrak{l}(2, \mathbb{R})$ of the conformal symmetry $\operatorname{Conf}\left(\mathbb{R}^{1}\right)$. Classical algebra (3.3) is characterized by the Casimir invariant $Q=K H_{g}-D^{2}$ that takes the value $Q=\frac{1}{4} g$. Last relation from (3.2) gives us solution to the equation of motion for the system (3.1),

$$
q(t)=\sqrt{2\left(a t^{2}+2 b t+c\right)},
$$

where real-valued constants $a, b$ and $c$ correspond to the values of the integrals $H_{g}, D$ and $K$, respectively, and thus are restricted by conditions $a>0, c>0$, and relation $a c-b^{2}=g / 4$ that guarantees positive values for the square root argument.

At the quantum level the noncommutativity of $D$ and $K$ with Hamiltonian $H_{g}$ in correspondence with the Poisson bracket relations (3.3) means that they do not generate symmetries in the usual sense of relating degenerate states, but rather they can be used to relate the states with different eigenvalues of the Hamiltonian operator.

Conformal symmetry (3.3) is a dynamical symmetry for the system (3.1), but it also is the isometry of $\mathrm{AdS}_{2}$. This underlies the interest to the model [2] in the context of the AdS/CFT correspondence and its diverse applications, in particular, related to the appearance of scale in nominally conformal theories [25].

Original system (3.1) has no scale, but the scale emerges in the theory via a mechanism described by de Alfaro, Fubini and Furlan [2]. This happens as follows. First relation in (3.3) and second relation in (3.2) reflect the fact that action (3.1) is explicitly invariant under the scale transformations $q \rightarrow e^{\alpha} q, t \rightarrow e^{2 \alpha} t, \alpha \in \mathbb{R}$, in accordance with which at the quantum level the dilatation operator generates the transformation $H_{g} \rightarrow e^{-2 \alpha} H_{g}$. Consequently, the Hamiltonian operator of the quantum version of the system (3.1) has a continuous spectrum in which there is no conformal invariant ground state. This can be related with the nature of the evolution coordinate $t$, which is not a good global coordinate on $\mathrm{AdS}_{2}$ [12].

\footnotetext{
${ }^{3}$ To treat integrals (3.2) as generators of the Lorentz algebra $\mathfrak{g} \mathfrak{o}(2,1)$ requires an introduction of a constant $\sigma$ with dimension of time (squared length) to make $H_{g}$ and $K$ dimensionless, $H_{g} \rightarrow \sigma H_{g}, K \rightarrow \sigma^{-1} K$, that, however, does not change the form of algebra (3.3).
}

To resolve this problem, one can consider the following change of the variables

$y(t)=\frac{q(t)}{\sqrt{u+v t+w t^{2}}}, \quad d \tau=\frac{d t}{u+v t+w t^{2}}$,

where $u>0, v$ and $w>0$ are real constants with dimensions $[u]=1,[v]=1 / t$ and $[w]=1 / t^{2}$. By replacement (3.5), action (3.1) transforms into

$$
\begin{aligned}
& \int \mathcal{L}\left(y, y^{\prime}\right) d \tau+\frac{1}{4} \int d \tau \frac{d}{d \tau}\left[(v+2 w t(\tau)) q^{2}(t(\tau))\right] \\
& =I[y]+I_{\text {surface }}
\end{aligned}
$$

where $\quad \mathcal{L}\left(y, y^{\prime}\right)=\frac{1}{2}\left(y^{\prime 2}-\frac{g}{y^{2}}-\omega^{2} y^{2}\right), \quad y^{\prime}=\frac{d y}{d \tau}, \quad$ and $\omega^{2}=\left(4 w u-v^{2}\right) / 4$. From the action $I[y]=\int \mathcal{L} d \tau$, we obtain the new time translation generator

$$
\mathcal{H}_{g}=\frac{1}{2}\left(p^{2}+\frac{g}{y^{2}}+\omega^{2} y^{2}\right), \quad p=y^{\prime}
$$

which is a compact $\mathfrak{g l}(2, \mathbb{R})$ generator when $\omega^{2}=$ $\left(4 w u-v^{2}\right) / 4>0$, whose quantum analog, like the quantum analog of $H_{g}$, has a spectrum restricted from below when $g \geq-1 / 4[53,54]$. The new evolution parameter $\tau=\frac{1}{\omega} \arctan \left(\frac{v+2 \omega t}{2 \omega}\right)$ varies in the finite interval $\left(-\frac{\pi}{2 \omega}, \frac{\pi}{2 \omega}\right)$, and new Hamiltonian (3.7) is conjugate to this good global time coordinate. In the context of black hole physics the AFF suggestion simply amounts to an improved choice of time coordinate [12,15]. As $\omega$ is a dimensionfull parameter, $[\omega]=[1 / t]$, (3.7) breaks the manifest scale invariance of the original system (3.1), and via such a basic mechanism the mass and length scales are introduced in holographic QCD (often referred to as "AdS/QCD") [25,26].

In spite of the introduced scale, the action of the new system is conformal invariant as we will see now. The dilatation generator $\mathcal{D}$ and the conformal transformation generator $\mathcal{K}$ associated with the action $I[y]$ are given by the explicitly depending on time $\tau$ integrals

$$
\begin{aligned}
\mathcal{D}=\frac{1}{2}\left(y p \cos (2 \omega \tau)+\left(2 \omega y^{2}-\mathcal{H}_{g} \omega^{-1}\right) \sin (2 \omega \tau)\right), \\
\mathcal{K}=\frac{1}{2}\left(y^{2} \cos (2 \omega \tau)-y p \omega^{-1} \sin (2 \omega \tau)\right. \\
\left.-\mathcal{H}_{g} \omega^{-2}(\cos (2 \omega \tau)-1)\right) .
\end{aligned}
$$

Via the Noether theorem, integrals (3.8) and (3.9) are related to the following infinitesimal symmetries of the action: 


$$
\begin{gathered}
\delta y=\epsilon_{\mathcal{D}} y \cos (2 \omega \tau), \\
\delta \tau=\epsilon_{\mathcal{D}} \omega^{-1} \sin (2 \omega \tau), \\
\delta(\mathcal{L} d \tau)=\frac{d}{d \tau}\left(-\epsilon_{\mathcal{D}} \omega y^{2} \sin (2 \omega t)\right) d \tau, \\
\delta y=-\epsilon_{\mathcal{K}} \omega^{-1} \sin (2 \omega \tau), \\
\delta \tau=\epsilon_{\mathcal{K}} \omega^{-2}(\cos (2 \omega \tau)-1), \\
\delta(\mathcal{L} d \tau)=\frac{d}{d \tau}\left(-\epsilon_{\mathcal{K}} \omega y^{2} \cos (2 \omega t)\right) d \tau .
\end{gathered}
$$

Integrals $\mathcal{H}_{g}, \mathcal{D}$ and $\mathcal{K}$ of the "regularized" AFF system generate the Newton-Hooke symmetry $[10,55,56]$

$$
\begin{aligned}
\left\{\mathcal{H}_{g}, \mathcal{D}\right\} & =-\left(\mathcal{H}_{g}-2 \omega^{2} \mathcal{K}\right), \\
\left\{\mathcal{H}_{g}, \mathcal{K}\right\} & =-2 \mathcal{D} \\
\{\mathcal{D}, \mathcal{K}\} & =-\mathcal{K},
\end{aligned}
$$

whose Casimir invariant is $\mathcal{Q}=\mathcal{K} \mathcal{H}_{g}-\mathcal{D}^{2}-\omega^{2} \mathcal{K}^{2}=$ $g / 4$. Using Eqs. (3.8) and (3.9), one can find solution to the equation of motion of the system (3.6),

$$
\begin{aligned}
y(\tau)= & \omega^{-1} \sqrt{\frac{2}{1+\sin ^{2}(2 \omega \tau)}} \\
& \times \sqrt{a \sin ^{2}(\omega \tau)+b \omega \sin (2 \omega \tau)+c \omega^{2} \cos (2 \omega \tau)},
\end{aligned}
$$

where $a>0, b$ and $c>0$ are constants corresponding to the values of the integrals $\mathcal{H}_{g}, \mathcal{D}$ and $\mathcal{K}$, respectively, and obeying the relation $a c-b^{2}-\omega^{2} c^{2}=g / 4$. From the explicit form of solution we see that it is periodic with the period $T=\pi / \omega$ not depending on the value of the coupling constant $^{4} g$. The finite interval in which the evolution parameter $\tau$ varies corresponds to the period of the motion of the system (3.6), and one can consider $\tau$ as the compact evolution parameter that takes values on the closed interval $\left[-\frac{\pi}{2 \omega}, \frac{\pi}{2 \omega}\right]$ with identified ends.

In the limit $\omega \rightarrow 0$, Hamiltonian $\mathcal{H}_{g}$ reduces to the Hamiltonian $H_{g}$ of the model (3.1), the integrals $\mathcal{D}$ and $\mathcal{K}$ reduce to the integrals $D$ and $K$, the algebra (3.12) takes the form (3.3), and bounded periodic solution (3.13) transforms into solution (3.4) for the system (3.1), which describes the unbounded motion of a particle in potential $g / q^{2}$. The symmetry transformations of the system (3.1) generated by $D$ and $K$ are recovered from (3.10) and (3.11). In particular, the nontrivial total time derivative in the last relation in (3.10) transforms in this limit into $\delta(L d t)=0$. This reflects the difference between manifest scale

\footnotetext{
${ }^{4}$ System given by Hamiltonian (3.7) is an isoperiodic deformation of the half-harmonic oscillator of frequency $\omega$ [57].
}

symmetry of the system (3.1) and more complicated, "hidden" form of the dilatation symmetry of the system given by Lagrangian $\mathcal{L}$. In application of the system (3.6) to the problem of confinement in $\mathrm{QCD}$, parameter $\omega$ introduces a mass scale, while the evolution parameter $\tau$ finds an interpretation as the difference of light-front times between events involving quarks and antiquarks in mesons [25].

To clarify further the relation between systems (3.1) and (3.6), we note that at $\tau=t=0$ we have $y(0)=q(0) / \sqrt{u}$, $y^{\prime}(0)=\sqrt{u} \dot{q}(0)-\frac{v q(0)}{2 \sqrt{u}}$, and therefore the Hamiltonian (3.7) becomes

$$
\mathcal{H}_{g}=u H_{g}-v D+w K .
$$

This shows that transformation (3.5) means in fact that a certain linear combination of $\mathfrak{g l}(2, \mathbb{R})$ generators is used as a new generator of time translations. To be more specific, we note that the $\mathfrak{g l}(2, \mathbb{R})$ algebra can be presented in the form of $\mathfrak{s} \mathfrak{o}(2,1)$ Lorentz algebra

$$
\left\{J^{\mu}, J^{\nu}\right\}=\epsilon^{\mu \nu \lambda} J_{\lambda},
$$

where $\mu, \nu, \lambda=0,1,2, J_{\mu}=\eta_{\mu \nu} J^{\nu}, \eta_{\mu \nu}=\operatorname{diag}(-1,1,1)$, and $\epsilon^{\mu \nu \lambda}$ is the Levi-Civita totally antisymmetric tensor, $\epsilon^{012}=1$. Comparison of (3.3) with (3.15) shows that the integrals $\sigma H_{g}, \sigma^{-1} K$ and $D$ of the system (3.1) can be identified, up to $\mathfrak{s} \mathfrak{o}(2,1)$ transformation, with generators $\left(J^{0}+J^{1}\right),\left(J^{0}-J^{1}\right)$ and $J^{2}$, and Casimir invariant $Q$ of algebra (3.3) corresponds to the Casimir element $-J^{\mu} J_{\mu}$ of algebra (3.15). Relation $-J^{\mu} J_{\mu}=g>0$ together with inequality $\left(J^{0}+J^{1}\right)>0$ describes the upper sheet of the two-sheeted hyperboloid in $(2+1)$ dimensional Minkowski space with coordinates $J^{1}, J^{2}, J^{0}$. According to such an identification, Hamiltonian $H_{g}$ corresponds to a noncompact $\mathfrak{g l}(2, \mathbb{R})$ generator of parabolic type [58,59] being a linear combination of generators of rotation and Lorentz boost transformations in $(2+1)$ dimensional Minkowski space. Putting then for simplicity $u=1$, $v=0, w=\sigma^{-2}$, we reduce (3.14) to the relation $\frac{1}{2} \sigma \mathcal{H}_{g}=$ $J^{0}$ that shows that Hamiltonian (3.7) is the generator of rotations in $(2+1)$-dimensional Minkowski space with coordinates $J^{\mu}$. According to Dirac [60,61], $H_{g}$ and $\mathcal{H}_{g}$ provide us with different forms of relativistic dynamics on the upper sheet of two-sheeted hyperboloid in $(2+1)$ dimensional Minkowski space, which by means of solutions (3.4) and (3.13) are projected to configuration spaces with coordinates $q$ and $y$, and are described by evolution parameters $t$ and $\tau$, respectively.

Changing the variable $y$ for $x=\sqrt{\omega} y$, and canonically quantizing (3.7), we obtain the Hamiltonian operator $(\hbar=1)$

$$
\hat{\mathcal{H}}_{g}=\frac{\omega}{2}\left(-\frac{d^{2}}{d x^{2}}+x^{2}+\frac{g}{x^{2}}\right), \quad x \in(0, \infty) .
$$


From now on, we assume that $g \geq-1 / 4$ to ensure that the spectrum of (3.16) is bounded from below [53,54]. In correspondence with this, one can set in (3.16) $g=$ $\nu(\nu+1)$ with $\nu \geq-1 / 2$. Following [3], as a domain of (3.16) we take $\left\{\psi \in L^{2}((0, \infty), d x) \mid \psi\left(0^{+}\right)=0\right\}$. As we will see, this guarantees that $\psi^{\prime}\left(0^{+}\right)=0$ for $\nu>0$, while $0<\left|\psi^{\prime}\left(0^{+}\right)\right|<\infty$ for $\nu=0$. We require additionally that $\left.\psi(x) \psi^{\prime}(x)\right|_{x=0^{+}}=0$ for $-1 / 2<\nu<0$, and $\left.\psi(x) \psi^{\prime}(x)\right|_{x=0^{+}}=$ $c \neq \infty$ when $\nu=-1 / 2$. In all the cases the specified properties of wave functions will guarantee that probability flux vanishes at $x=0^{+}$. The specified domain corresponds to (essentially) self-adjoint nature of the operator (3.16) in the case of $\nu \geq 1 / 2$, and to a special case $\theta=\pi / 2$ of one-parametric, $\theta \in[0, \pi]$, families of self-adjoint extensions of $\hat{\mathcal{H}}_{g}$ in the cases of $-1 / 2<\nu<1 / 2$ and $\nu=-1 / 2$. This special case is the unique value of $\theta$ for which the spectrum of (3.16) with $-1 / 4 \leq g<3 / 4$ is equidistant similarly to the case of $g \geq 3 / 4$. For the detailed discussion of the issue of self-adjoint extensions of the operator (3.16) we refer to $[54,62,63]$. In the next section we shall see that the specified quantum Hamiltonian operator (3.16) corresponds, in accordance with the described above classical picture, to the so called discrete type representation $\mathcal{D}_{\alpha}^{+}$of the $\mathfrak{g l}(2, \mathbb{R})$ algebra characterized by the value of the Casimir operator $\hat{J}^{\mu} \hat{J}_{\mu}=-\alpha(\alpha-1)$, in which the compact generator $\hat{J}^{0}$ takes positive discrete values $j_{n}^{0}=n+\alpha$, $n=0,1, \ldots$, with $\alpha=\frac{1}{2} \nu+\frac{3}{4} \geq \frac{1}{2}$ [59].

In what follows, we identify new, not described earlier in the literature, finite discrete symmetries of the Schrödinger equation with Hamiltonian operator of the regularized AFF model. We show that these finite discrete symmetries, described by the Klein four-group, are nontrivially encoded in the conformal symmetry of the AFF model. We also find that this discrete symmetry has peculiarities in the case of half-integer values of the parameter $\nu$, and, in particular, at $\nu=-1 / 2$. The value $\nu=-1 / 2$, as we will see, also is special in superextensions of the AFF model, where the transformations of the Klein four-group appear as automorphisms of the superconformal $\mathfrak{o} \mathfrak{g} \mathfrak{p}(2,2)$ symmetry and relate (mutually map) the corresponding systems with exact and spontaneously broken $\mathcal{N}=2$ Poincaré supersymmetries. Then we use these discrete symmetries together with the conformal symmetry to generate, by means of Darboux transformations, infinite families of new, exactly solvable quantum systems with equidistant spectra containing arbitrary number of gaps and described by symmetries of the type of finite $W$ algebras, which represent nonlinearly deformed and extended forms of the conformal $\mathfrak{g l}(2, \mathbb{R})$ algebra.

\section{SYMMETRIES OF THE QUANTUM AFF MODEL AND ITS $\mathcal{N}=2$ SUPEREXTENSIONS}

In this section, we discuss some aspects of symmetry of the quantum AFF model given by the Hamiltonian operator (3.16). Namely, we show that the corresponding Schrödinger equation has a finite discrete symmetry, which is broken in general case and is encoded in the "fine" structure of conformal symmetry of the system. It also will be shown that in the case of half-integer values of the parameter $\nu$, the finite discrete symmetry has some peculiarities related to the structure of eigenstates and eigenvalues of the quantum Hamiltonian (3.16). These peculiarities are manifested in a spectral symmetry realized in the form of a "departure from the Hilbert's hotel" mechanism and in appearance of Jordan states in kernels of the ladder operators being noncompact generators of conformal symmetry. We also discuss here the effect of discrete group on superconformal symmetry of $\mathcal{N}=2$ super-extensions of the AFF system.

Consider the time-dependent Schrödinger equation of the quantum system (3.16),

$$
i \frac{\partial \Psi_{\nu}(x, t)}{\partial t}=\mathcal{H}_{\nu} \Psi_{\nu}(x, t),
$$

with

$$
\mathcal{H}_{\nu}=-\frac{d^{2}}{d x^{2}}+x^{2}+\frac{\nu(\nu+1)}{x^{2}} .
$$

Here, the operator $\mathcal{H}_{\nu}$ corresponds to (3.16) with $g(\nu)=$ $\nu(\nu+1), \nu \geq-1 / 4$, and $\omega=2$. Solutions of Eq. (4.1) in the form of stationary states $\Psi_{\nu}(x, t)=e^{-i t \lambda_{\nu}} \psi_{\nu}(x)$ are given in terms of the well known physical eigenstates of $\mathcal{H}_{\nu}$ represented by normalized wave functions satisfying the boundary conditions at $x=0$,

$$
\begin{aligned}
\psi_{\nu, n}(x) & =\sqrt{\frac{n !}{\Gamma(n+\nu+3 / 2)}} x^{\nu+1} L_{n}^{(\nu+1 / 2)}\left(x^{2}\right) e^{-x^{2} / 2}, \\
E_{\nu, n} & =2 \nu+4 n+3,
\end{aligned}
$$

where $E_{\nu, n}$ are the eigenvalues, $n=0,1, \ldots$, and

$$
L_{n}^{(\alpha)}(x)=\sum_{k=0}^{n} \frac{\Gamma(n+\alpha+1)}{\Gamma(k+\alpha+1)} \frac{(-x)^{k}}{k !(n-k) !}
$$

are the generalized Laguerre polynomials.

Then the problem $\mathcal{H}_{\nu} \psi_{\nu}=\lambda_{\nu} \psi_{\nu}$ fits with (2.1), but to generate new exactly solvable systems and identify their ladder operators and the associated deformed conformal symmetries we should not be limited just by considering physical states. In subsequent sections we will see how nonphysical states obtained via the application of certain discrete symmetry group transformations play an important role in the structure of the quantum conformal algebra generators of the AFF model and its $\mathfrak{o} \mathfrak{g} \mathfrak{p}(2 \mid 2)$ superconformal extensions, as well as in the construction of new systems and their hidden symmetries. 


\section{A. The Klein four-group as a Schrödinger equation symmetry}

Parametrizing the coupling constant in parabolic form $g=\nu(\nu+1)$, which is symmetric with respect to $\nu=-\frac{1}{2}$, we artificially induce the invariance of the Eq. (4.1) with respect to the transformation $\rho_{1}: \nu \rightarrow-\nu-1$. Equation (4.1) is also invariant with respect to the transformation $\rho_{2}$ : $(x, t) \rightarrow(i x,-t)$. These two transformations generate the Klein four-group as a symmetry of Eq. (4.1): $K_{4} \simeq \mathbb{Z}_{2} \times$ $\mathbb{Z}_{2}=\left(1, \rho_{1}, \rho_{2}, \rho_{1} \rho_{2}=\rho_{2} \rho_{1}\right)$, where each element is its own inverse. At the level of the stationary Schrödinger equation, the action of $\rho_{2}$ reduces to the transformation $\rho_{2}:\left(x, E_{\nu, n}\right) \rightarrow\left(i x,-E_{\nu, n}\right)$, which means that $\rho_{2}$ is a completely broken $\mathbb{Z}_{2}$ symmetry, for which the transformed eigenstates $\rho_{2}\left(\psi_{\nu, n}\right)=\psi_{\nu, n}(i x)$ with eigenvalues $-E_{\nu, n}$ are nonphysical solutions. The transformation $\rho_{1}$ at the same level of the stationary Schrödinger equation implies that the energy eigenvalues change as $E_{\nu, n} \rightarrow \rho_{1}\left(E_{\nu, n}\right)=$ $E_{-\nu-1, n}=4 n-2 \nu+1$. The difference between the original energy level and the transformed one is $E_{\nu, n}-E_{-\nu-1, n}=$ $\Delta E \cdot(\nu+1 / 2)$, where $\Delta E=4$ is the distance between two consecutive levels. So, if we take $\nu=\ell-1 / 2$ with $\ell=0,1, \ldots$, we obtain $\rho_{1}\left(E_{\ell-1 / 2, n}\right)=E_{\ell-1 / 2, n-\ell}$, and find that physical energy levels with $n \geq \ell$ are transformed into physical energy levels but lowered by $4 \ell$. Under the action of $\rho_{1}$, the eigenstates (4.3) are transformed into the functions

$$
\begin{aligned}
\rho_{1}\left(\psi_{\nu, n}\right) & =\sqrt{\frac{n !}{\Gamma(n-\nu+1 / 2)}} x^{-\nu} L_{n}^{(-\nu-1 / 2)}\left(x^{2}\right) e^{-x^{2} / 2} \\
& :=\psi_{-\nu-1, n} .
\end{aligned}
$$

In the case of $\nu \neq \ell-1 / 2$, functions (4.5) do not satisfy boundary condition at $x=0$ because of the presence of the factor $x^{-\nu}$, and they are nonphysical, formal eigenstates of $\mathcal{H}_{\nu}$. The case of $\nu=\ell-1 / 2$ requires, however, a separate consideration. To analyze this case, we observe that

$$
\rho_{1}\left(\psi_{\ell-1 / 2, n}\right)=\sqrt{\frac{n !}{\Gamma(n-\ell+1)}} x^{-\ell+1 / 2} L_{n}^{(-\ell)}\left(x^{2}\right) e^{-x^{2} / 2} .
$$

Due to the poles of Gamma function, this expression vanishes when $n<\ell$, i.e., $\rho_{1}$ annihilates the first $\ell$ eigenstates of the system. On the other hand, the identity

$$
\frac{(-\eta)^{m}}{m !} L_{n}^{(m-n)}(\eta)=\frac{(-\eta)^{n}}{n !} L_{m}^{(n-m)}(\eta)
$$

with integer $m$ and $n$, which follows from (4.4), allows us to write $\rho_{1}\left(\psi_{\ell-1 / 2, n}\right)=(-1)^{\ell} \psi_{\ell-1 / 2, n-\ell}$ when $n \geq \ell$, and this is coherent with the change of the energy eigenvalues under application to them of transformation $\rho_{1}$. In conclusion, $\rho_{1}$ corresponds to a symmetry which is just the identity operator when $\ell=0$, while for $\ell \geq 1$ this symmetry annihilates the $\ell$ lowest physical eigenstates, but restores them by acting on the higher eigenstates. ${ }^{5}$ From this point of view, in the case of half-integer $\nu$, transformation $\rho_{1}$ does not produce anything new. Nevertheless, we can also construct a finite set of nonphysical solutions of the same nonphysical nature as in (4.5) given by the functions

$$
\begin{aligned}
\psi_{-\ell-1 / 2, k} & :=\rho_{1}\left(\sqrt{\frac{\Gamma(k+l+1)}{k !}} \psi_{\ell-1 / 2, k}\right) \\
& =x^{-\ell+1 / 2} L_{n}^{(-\ell)}\left(x^{2}\right) e^{-x^{2} / 2}, \\
k & =0, \ldots, \ell-1,
\end{aligned}
$$

singular at $x=0$, whose corresponding eigenvalues are $E_{-\ell-1 / 2, n}=4 n-2 \ell+2$.

We note that the combined transformation $\rho_{1} \rho_{2}\left(\psi_{\nu, n}\right)$ always produces nonphysical solutions for all values of $\nu$ due to the presence of $\rho_{2}$. Wave eigenfunctions transformed by the $K_{4}$ generators $\rho_{2}$ and $\rho_{1} \rho_{2}$ diverge exponentially at infinity, and for the following consideration it is convenient to introduce a special common notation for them: $\psi_{r(\nu),-n}(x)=\psi_{r(\nu), n}(i x)$, where $r(\nu)=\nu$ corresponds to application of $\rho_{2}$, and $r(\nu)=-\nu-1$ corresponds to application of $\rho_{1} \rho_{2}$ to $\psi_{\nu, n}(x)$. In the same way, we introduce a common notation for physical eigenstates and nonphysical eigenfunctions exponentially disappearing at infinity: $\psi_{r(\nu), n}(x)$, where $r(\nu)=\nu$ corresponds to eigenstates (4.3), and $r(\nu)=-\nu-1$ corresponds to nonphysical eigenfunctions (4.5) or (4.8) when $\nu=\ell-1 / 2$. In the case of $\quad \nu=\ell-1 / 2, \quad \ell \geq 1$, we have $E_{-\ell-1 / 2, \ell-n-1}=$ $-E_{-\ell-1 / 2, n}$ for $n<\ell$, and one finds that (4.8) and their partners in the sense of Eq. (2.7) are related with nonphysical eigenstates produced by $\rho_{2}$ and their partners,

$$
\begin{aligned}
& \psi_{-\ell-1 / 2, \ell-1-n} \propto \tilde{\psi}_{-\ell-1 / 2,-n}, \\
& \tilde{\psi}_{-\ell-1 / 2, n} \propto \psi_{-\ell-1 / 2,-\ell+1-n} .
\end{aligned}
$$

\section{B. Conformal symmetry and ladder operators}

In this subsection, we explore the quantum conformal symmetry of the model from the perspective of the discrete Klein four-group symmetry.

Hamiltonian (4.2) is the compact generator of the dynamical conformal symmetry of the AFF model, which together with the second order differential operators

${ }^{5}$ This is similar to a picture of a Hilbert's hotel under departure of clients from first $\ell$ rooms with numbers $n=0, \ldots, \ell-1$ with simultaneous translation of the clients from rooms with numbers $n=\ell, \ell+1, \ldots$, into the rooms with numbers $n-\ell$. Note that the power $\left(\mathcal{C}^{-}\right)^{\ell}$ of lowering generator of conformal symmetry (4.10) with $\nu=\ell-\frac{1}{2}$ acts on physical eigenstates in a way similar to $\rho_{1}$, but violating normalization of the states, see Eq. (4.14). 


$$
\mathcal{C}_{\nu}^{-}=-\left(\frac{d}{d x}+x\right)^{2}+\frac{\nu(\nu+1)}{x^{2}}, \quad \mathcal{C}_{\nu}^{+}=\left(\mathcal{C}_{\nu}^{-}\right)^{\dagger}
$$

$$
\mathcal{C}_{\nu}^{ \pm} \psi_{r(\nu), n}=\sqrt{\left(E_{r(\nu), n} \pm 2 \nu \pm 3\right)\left(E_{r(\nu), n} \pm 2 \nu \mp 1\right)} \psi_{r(\nu), n \pm 1},
$$

satisfies the commutation relations of the $\mathfrak{g l}(2, \mathbb{R})$ algebra,

$$
\left[\mathcal{H}_{\nu}, \mathcal{C}_{\nu}^{ \pm}\right]= \pm 4 \mathcal{C}_{\nu}^{ \pm}, \quad\left[\mathcal{C}_{\nu}^{-}, \mathcal{C}_{\nu}^{+}\right]=8 \mathcal{H}_{\nu}
$$

whose Casimir invariant is given by $\mathcal{C}=\left(\mathcal{H}_{\nu}\right)^{2}-$ $\frac{1}{2}\left(\mathcal{C}_{\nu}^{+} \mathcal{C}_{\nu}^{-}+\mathcal{C}_{\nu}^{-} \mathcal{C}_{\nu}^{+}\right)=4 \nu(\nu+1)-3$. Taking linear combinations $\mathcal{J}^{0}:=\frac{1}{4} \mathcal{H}_{\nu}, \mathcal{J}^{1}:=\frac{1}{8}\left(\mathcal{C}_{\nu}^{+}+\mathcal{C}_{\nu}^{-}\right), \mathcal{J}^{2}:=\frac{i}{8}\left(\mathcal{C}_{\nu}^{-}-\mathcal{C}_{\nu}^{+}\right)$, one finds that they satisfy the quantum analog of the classical Lorentz algebra (3.15). The rescaled Casimir operator $-\frac{1}{16} \mathcal{C}$ represented in their terms reduces to $\mathcal{J}^{\mu} \mathcal{J}_{\mu}=-\alpha(\alpha-1)$ with $\alpha=\frac{1}{2} \nu+\frac{3}{4}$, and as it was indicated at the end of the previous section, eigenvalues of $\mathcal{J}^{0}$ are $j^{0}=n+\alpha, n=0,1, \ldots$. We note here that the linear combinations $\mathcal{J}^{0}-\mathcal{J}^{1}$ and $\mathcal{J}^{0}+\mathcal{J}^{1}$ are the operators $\frac{1}{2} x^{2}$ and $\frac{1}{2}\left(-\frac{d^{2}}{d x^{2}}+\frac{\nu(\nu+1)}{x^{2}}\right)$, which are the quantum analogs of the integrals $K$ and $H_{g}$ defined in (3.2) for the model (3.1) with $q$ changed for $x$ and $t=0$.

Coefficient 4 in the first commutator in (4.11) is the distance between the consecutive energy levels of the AFF system, and by means of the unitary transformation

$$
\mathcal{C}_{\nu}^{ \pm} \rightarrow \mathcal{C}_{\nu}^{ \pm}(t)=e^{-i t \mathcal{H}_{\nu}} \mathcal{C}_{\nu}^{ \pm} e^{i t \mathcal{H}_{\nu}}=e^{\mp i 4 t} \mathcal{C}_{\nu}^{ \pm},
$$

we obtain two dynamical integrals of motion, now in the sense of the Heisenberg equation $\frac{d}{d t} A=\frac{\partial A}{\partial t}-i\left[A, \mathcal{H}_{\nu}\right]=0$. Their linear Hermitian combinations

$\mathcal{D}(t)=i \frac{\mathcal{C}_{\nu}^{-}(t)-\mathcal{C}_{\nu}^{+}(t)}{8}, \quad \mathcal{K}(t)=\frac{2 \mathcal{H}_{\nu}-\mathcal{C}_{\nu}^{-}(t)-\mathcal{C}_{\nu}^{+}(t)}{16}$,

are the quantum analogs of the generators of the NewtonHooke symmetry (3.8) with $\omega=2$.

According to (4.11), operators $\mathcal{C}_{\nu}^{ \pm}$are the ladder operators of the quantum AFF system described by the Hamiltonian $\mathcal{H}_{\nu}$. The generator $\rho_{1}$ of the discrete $K_{4}$ group acts identically on generators of the conformal symmetry, $\rho_{1}\left(\mathcal{H}_{\nu}\right)=\mathcal{H}_{\nu}, \rho_{1}\left(\mathcal{C}_{\nu}^{ \pm}\right)=\mathcal{C}_{\nu}^{ \pm}$, while $\rho_{2}\left(\mathcal{H}_{\nu}\right)=-\mathcal{H}_{\nu}, \rho_{2}\left(\mathcal{C}_{\nu}^{ \pm}\right)=$ $-\mathcal{C}_{\nu}^{\mp}$. In correspondence with this, $\rho_{2}$ is the automorphism of the $\mathfrak{g} \mathfrak{l}(2, \mathbb{R})$ algebra which transforms the unitary irreducible representation $\mathcal{D}_{\alpha}^{+}$of the system (4.2) restricted from below, $j^{0}=n+\alpha, \alpha=\frac{1}{2} \nu+\frac{3}{4} \geq \frac{1}{2}$, into the unitary irreducible representation $\mathcal{D}_{\alpha}^{-}$of $\mathfrak{g l}(2, \mathbb{R})$ restricted from above, $j^{0}=-(n+\alpha), n=0,1, \ldots$ [59].

The ladder operators act on physical eigenstates and nonphysical states generated from them by transformations of the $K_{4}$ group as follows:

$$
\begin{aligned}
& \mathcal{C}_{\nu}^{ \pm} \psi_{r(\nu),-n} \\
& \quad=-\sqrt{\left(E_{r(\nu), n} \pm 2 \nu \pm 3\right)\left(E_{r(\nu), n} \pm 2 \nu \mp 1\right)} \psi_{r(\nu),-(n \mp 1)} .
\end{aligned}
$$

The coefficients in (4.14) and (4.15) vanish when the ladder operators act on the states from their corresponding kernels, which in the case of $\nu>-1 / 2$ are given by

$$
\begin{aligned}
\operatorname{ker} \mathcal{C}_{\nu}^{-} & =\operatorname{span}\left\{\psi_{\nu, 0}, \psi_{-\nu-1,0}\right\}, \\
\operatorname{ker} \mathcal{C}_{\nu}^{+} & =\operatorname{span}\left\{\psi_{\nu,-0}, \psi_{-\nu-1,-0}\right\} .
\end{aligned}
$$

In the case of $\nu=-1 / 2$, the kernels of the ladder operators $\mathcal{C}_{-1 / 2}^{ \pm}$are similar to (4.16) but with the states $\psi_{-\nu-1,0}$ and $\psi_{-\nu-1,-0}$ there changed, respectively, for the Jordan states

$$
\begin{aligned}
\Omega_{-1 / 2,0} & =\left(a-\frac{1}{2} \ln x\right) \psi_{-1 / 2,0}, \\
\Omega_{-1 / 2,-0} & =\left(b-\frac{1}{2} \ln x\right) \psi_{-1 / 2,-0},
\end{aligned}
$$

where $a$ and $b$ are constants.

In the context of the Darboux transformations, the first equation in (4.11) can be written in the equivalent form $\mathcal{C}_{\nu}^{\mp} \mathcal{H}_{\nu}=\left(\mathcal{H}_{\nu} \pm 4\right) \mathcal{C}_{\nu}^{\mp}$, which means that $\mathcal{C}_{\nu}^{ \pm}$intertwine the system $\mathcal{H}_{\nu}$ with itself but shifted for additive constants $\mp 4$. Then Eq. (4.16) indicates that the second order differential operators $-\mathcal{C}_{\nu}^{ \pm}$are generated by the choice of the seed states $\left(\psi_{\nu, \mp 0}, \psi_{-\nu-1, \pm 0}\right)$, and by means of Eq. (2.3) we can write the equalities

$$
\mathcal{C}_{\nu}^{\mp} \phi_{r(\nu), z}=-\frac{W\left(\psi_{\nu, \pm 0}, \psi_{-\nu-1, \pm 0}, \phi_{r(\nu), z}\right)}{W\left(\psi_{\nu, \pm 0}, \psi_{-\nu-1, \pm 0}\right)},
$$

where $\phi_{r(\nu), z}$ with $z= \pm n, n \in \mathbb{N}$, corresponds to an eigenstate or a Jordan state of $\mathcal{H}_{\nu}$. The Wronskian form of these equalities is useful to find the action of the ladder operators on the states $\tilde{\psi}_{r(\nu), \pm 0}$ and $\breve{\Omega}_{-1 / 2,0}$. Using Eqs. (2.9) and (2.19), and equalities

$$
\begin{aligned}
W\left(\psi_{\nu, \pm 0}, \psi_{-\nu-1, \pm 0}\right) & =-(2 \nu+1) e^{\mp x^{2}}, \\
W\left(\psi_{-1 / 2, \pm 0}, \Omega_{-1 / 2, \pm 0}\right) & =e^{\mp x^{2}},
\end{aligned}
$$

one can find that

$\mathcal{C}_{\nu}^{-} \tilde{\psi}_{r(\nu), 0} \propto \psi_{r(-\nu-1),-0}, \quad \mathcal{C}_{\nu}^{+} \tilde{\psi}_{r(\nu),-0} \propto \psi_{r(-\nu-1), 0}$, 
(a) Case $\nu=\ell-1 / 2, \quad \ell=1,2, \ldots$.

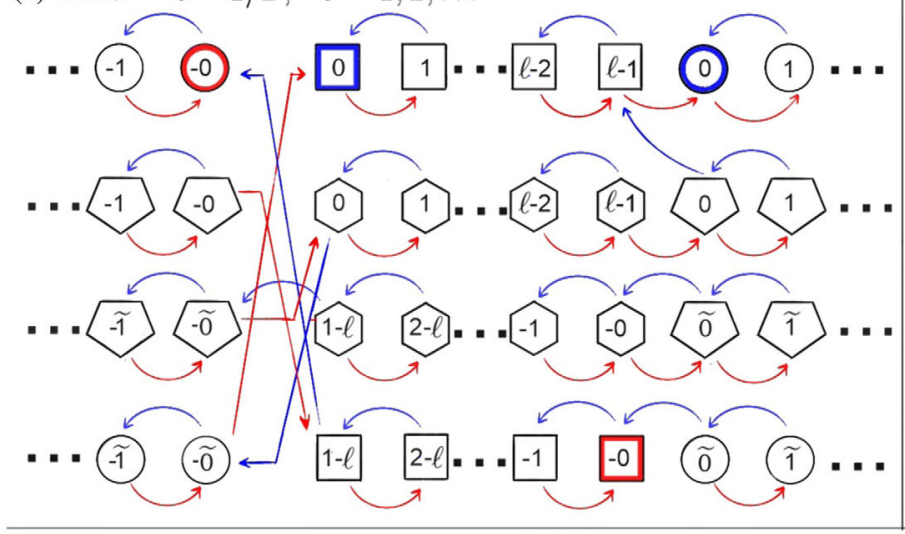

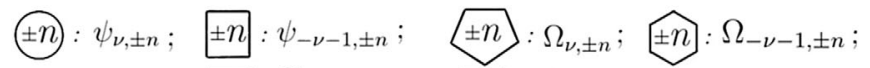

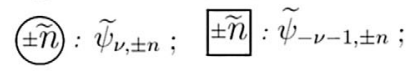

$\pm \widetilde{n}: \breve{\Omega}_{\nu, \pm n}$

$\widehat{ \pm n}: \Omega_{-\nu-1, \pm n} ;$
$\mathcal{C}_{\nu}^{-}:$

$\mathcal{C}_{\nu}^{+}:$

(b) Case $\nu \neq \ell-1 / 2$

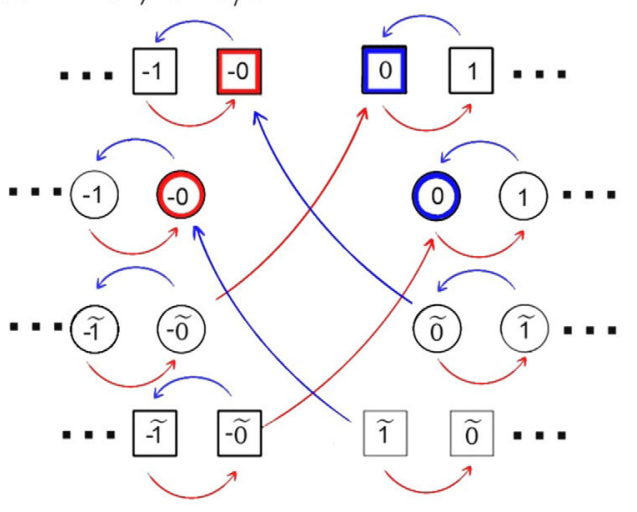

FIG. 1. The action of the ladder operators in dependence on the value of $\nu$. Diagram (a) illustrates the case of half-integer $\nu=\ell-1 / 2$ with $\ell=1, \ldots$, where it is shown how Jordan states can be related to eigenstates by the action of $\mathcal{C}_{\nu}^{ \pm}$. Diagram (b) corresponds to nonhalf-integer values of $\nu$. In (c), it is indicated how the case with $\nu=-1 / 2$ can be obtained from (b) by changing the corresponding states. The shapes with borders highlighted in blue (red) represent the states annihilated by $\mathcal{C}_{\nu}^{-}\left(\mathcal{C}_{\nu}^{+}\right)$.

$\mathcal{C}_{-1 / 2}^{\mp} \tilde{\psi}_{-1 / 2, \pm 0} \propto \Omega_{-1 / 2, \mp 0}, \quad \mathcal{C}_{-1 / 2}^{\mp} \breve{\Omega}_{-1 / 2, \pm 0} \propto \psi_{-1 / 2, \mp 0}$.

Returning to the issue of Jordan states, we realize that they have appeared in the systems with half-integer $\nu$, but let us consider first the general case. For this, we use (2.15) and the first relation in (4.11) to prove the relations

$$
\Omega_{r(\nu), \pm n} \propto\left(\mathcal{C}_{\nu}^{ \pm}\right)^{n} \Omega_{r(\nu), \pm 0}, \quad \breve{\Omega}_{r(\nu), \pm n} \propto\left(\mathcal{C}_{\nu}^{ \pm}\right)^{n} \breve{\Omega}_{r(\nu), \pm 0}
$$

Thus, the ladder operators act in a similar way as they act on eigenstates of $\mathcal{H}_{\nu}$, but with a difference when $n=0$. When $\nu \neq-1 / 2$, we obtain the relations $\mathcal{C}_{\nu}^{ \pm} \Omega_{r(\nu), \mp 0} \propto$ $\tilde{\psi}_{r(-\nu-1), \pm 0}$ and $\mathcal{C}_{\nu}^{ \pm} \breve{\Omega}_{r(\nu), \mp 0} \propto \Omega_{r(-\nu-1), \pm 0}$. Due to (4.9) one can make the identification $\breve{\Omega}_{-\ell-1 / 2, \pm 0}=$ $\Omega_{-\ell-1 / 2, \mp(\ell-1)}$, so in the half-integer case $\nu=\ell-1 / 2$ with $\ell \geq 1$ we obtain

$$
\begin{aligned}
& \mathcal{C}_{\ell-1 / 2}^{ \pm} \Omega_{\ell-1 / 2, \mp 0} \propto \psi_{-\ell-1 / 2, \mp(\ell-1)}, \\
& \mathcal{C}_{\ell-1 / 2}^{ \pm} \Omega_{-\ell-1 / 2, \mp 0} \propto \psi_{\ell-1 / 2, \mp(\ell-1)} .
\end{aligned}
$$

Acting on these relations by $\left(\mathcal{C}_{\ell-1 / 2}^{ \pm}\right)^{\ell}$, we obtain zero, and conclude that

$$
\begin{aligned}
\operatorname{ker}\left(\mathcal{C}_{\ell-1 / 2}^{ \pm}\right)^{\ell+k}= & \operatorname{span}\left\{\psi_{\ell-1 / 2, \mp 0}, \ldots, \psi_{\ell-1 / 2, \mp(\ell+k-1)},\right. \\
& \psi_{-(\ell-1 / 2)-1, \mp 0}, \ldots, \psi_{-(\ell-1 / 2)-1, \mp(\ell-1)}, \\
& \left.\Omega_{\ell-1 / 2, \mp 0}, \ldots, \Omega_{\ell-1 / 2, \mp(k-1)}\right\}
\end{aligned}
$$

for $k=1,2, \ldots$. The whole picture is summarized in Fig. 1.

\section{Superconformal symmetry and the Klein four-group}

Here, we inspect the action of the transformations of the Klein four-group on a supersymmetric extension of the AFF model.

One can take two different ways to produce two different supersymmetric extensions of the AFF model by taking two different Darboux transformations of the first order based on the choice of the seed states $\psi_{\nu, 0}$ and $\psi_{\nu,-0}$. By means of Eq. (2.4) with $n=1$ we obtain two corresponding pairs of Hermitian conjugate intertwining operators,

$\begin{array}{ll}A_{\nu}^{-}=\frac{d}{d x}+x-\frac{\nu+1}{x}, & A_{\nu}^{+}=\left(A_{\nu}^{-}\right)^{\dagger}, \\ B_{\nu}^{-}=\frac{d}{d x}-x-\frac{\nu+1}{x}, & B_{\nu}^{+}=\left(B_{\nu}^{-}\right)^{\dagger} .\end{array}$

These operators link the systems $\mathcal{H}_{\nu}$ and $\mathcal{H}_{\nu+1}$ by the intertwining relations of the form (2.5),

$$
A_{\nu}^{-} \mathcal{H}_{\nu}=\left(\mathcal{H}_{\nu+1}+2\right) A_{\nu}^{-}, \quad A_{\nu}^{+} \mathcal{H}_{\nu+1}=\left(\mathcal{H}_{\nu}-2\right) A_{\nu}^{+},
$$

$$
B_{\nu}^{-} \mathcal{H}_{\nu}=\left(\mathcal{H}_{\nu+1}-2\right) B_{\nu}^{-}, \quad B_{\nu}^{+} \mathcal{H}_{\nu+1}=\left(\mathcal{H}_{\nu}+2\right) B_{\nu}^{+}
$$

Let us note here that if we choose, instead, nonphysical eigenstates $\psi_{-\nu-1,0}$ and $\psi_{-\nu-1,-0}$ as the seed states, we generate the operators $-A_{\nu-1}^{+}$and $-B_{\nu-1}^{+}$, respectively. These operators relate the system $\mathcal{H}_{\nu}$ with $\mathcal{H}_{\nu-1}$ by 
intertwining relations of the form (4.26) and (4.27) with $\nu$ shifted by minus one.

From the point of view of the Klein four-group, the states $\psi_{\nu, 0}$ and $\psi_{\nu,-0}$ are related by transformation $\rho_{2}$, while the states $\psi_{\nu, \pm 0}$ and $\psi_{-\nu-1, \pm 0}$ are mutually transformed by $\rho_{1}$. As a consequence, the application of transformations $\rho_{1}$ and $\rho_{2}$ to the intertwining operators gives

$$
\begin{array}{r}
\rho_{1}\left(A_{\nu}^{\mp}\right)=-B_{\nu-1}^{ \pm}, \quad \rho_{1}\left(B_{\nu}^{\mp}\right)=-A_{\nu-1}^{ \pm}, \\
\rho_{2}\left(A_{\nu}^{ \pm}\right)=-i B_{\nu}^{ \pm}, \quad \rho_{2}\left(B_{\nu}^{ \pm}\right)=-i A_{\nu}^{ \pm} .
\end{array}
$$

These relations are valid for $\nu>-1 / 2$. At $\nu=-1 / 2$ the transformation $\rho_{1}$ reduces to the identity.

We can construct now two different $\mathcal{N}=2$ superHamiltonians

$$
\begin{gathered}
\mathcal{H}_{\nu}^{e}=\left(\begin{array}{cc}
A_{\nu}^{-} A_{\nu}^{+}=\mathcal{H}_{\nu+1}-2 \nu-1 & 0 \\
0 & A_{\nu}^{+} A_{\nu}^{-}=\mathcal{H}_{\nu}-2 \nu-3
\end{array}\right), \\
\mathcal{H}_{\nu}^{b}=\left(\begin{array}{cc}
B_{\nu}^{-} B_{\nu}^{+}=\mathcal{H}_{\nu+1}+2 \nu+1 & 0 \\
0 & B_{\nu}^{+} B_{\nu}^{-}=\mathcal{H}_{\nu}+2 \nu+3
\end{array}\right),
\end{gathered}
$$

where the indexes $e$ and $b$ refer to the exact and spontaneously broken $\mathcal{N}=2$ Poincaré supersymmetries described by the corresponding super-Hamiltonians. The operator (4.30) has an equidistant spectrum given by eigenvalues $\mathcal{E}_{n}=4 n, n=0,1, \ldots$, where $n=0$ corresponds to the nondegenerate ground state $\left(0, \psi_{\nu, 0}\right)^{t}$ of zero energy, while all the energy levels with $n \geq 1$ are doubly degenerate. The Hamiltonian (4.31) has eigenvalues $\mathcal{E}_{n}=4 n+4 \nu+6$, each of which is doubly degenerate, and two ground states with energy $\mathcal{E}_{0}=4 \nu+6>0$ are $\Psi_{0}^{(+)}=$ $\left(B_{\nu}^{-} \psi_{\nu-1,0}, \psi_{\nu-1,0}\right)^{t}$ and $\Psi_{0}^{(-)}=\sigma_{3} \Psi_{0}^{(+)}$.

System (4.30) is described by the $\mathfrak{o} \mathfrak{g} \mathfrak{p}(2,2)$ superconformal dynamical symmetry generated by the even, $\mathcal{H}_{\nu}^{e}$, $\mathcal{R}_{\nu}=\frac{1}{4}\left(\mathcal{H}_{\nu}^{e}-\mathcal{H}_{\nu}^{b}\right)=\frac{\sigma_{3}}{2}-(\nu+1) \mathbb{I}, \mathcal{G}_{\nu}^{ \pm}$, and odd, $\mathcal{Q}_{\nu}^{a}, \mathcal{S}_{\nu}^{a}$, $a=1,2$, operators, where $\mathbb{I}$ is the unit $2 \times 2$ matrix,

$$
\begin{gathered}
\mathcal{G}_{\nu}^{ \pm}=\left(\begin{array}{cc}
\mathcal{C}_{\nu+1}^{ \pm} & 0 \\
0 & \mathcal{C}_{\nu}^{ \pm}
\end{array}\right), \\
\mathcal{Q}_{\nu}^{1}=\left(\begin{array}{cc}
0 & A_{\nu}^{-} \\
A_{\nu}^{+} & 0
\end{array}\right), \quad \mathcal{S}_{\nu}^{1}=\left(\begin{array}{cc}
0 & B_{\nu}^{-} \\
B_{\nu}^{+} & 0
\end{array}\right), \\
\mathcal{Q}_{\nu}^{2}=i \sigma_{3} \mathcal{Q}_{\nu}^{1}, \quad \mathcal{S}_{\nu}^{2}=i \sigma_{3} \mathcal{S}_{\nu}^{1} .
\end{gathered}
$$

Here $\mathcal{Q}_{\nu}^{a}$ are the supercharges of the system, which annihilate the ground state, i.e., the system indeed is in the phase of unbroken $\mathcal{N}=2$ Poincaré supersymmetry. The Lie superalgebraic relations

$$
\begin{gathered}
{\left[\mathcal{H}_{\nu}^{e}, \mathcal{R}_{\nu}\right]=\left[\mathcal{H}_{\nu}^{e}, \mathcal{Q}_{\nu}^{a}\right]=0} \\
{\left[\mathcal{H}_{\nu}^{e}, \mathcal{G}_{\nu}^{ \pm}\right]= \pm 4 \mathcal{G}_{\nu}^{ \pm}, \quad\left[\mathcal{G}_{\nu}^{-}, \mathcal{G}_{\nu}^{+}\right]=8 \mathcal{H}_{\nu}^{e}-16 \mathcal{R}_{\nu},} \\
{\left[\mathcal{H}_{\nu}^{e}, \mathcal{S}_{\nu}^{a}\right]=-4 i \epsilon^{a b} \mathcal{S}_{\nu}^{b}, \quad\left[\mathcal{R}_{\nu}, \mathcal{Q}_{\nu}^{a}\right]=-i \epsilon^{a b} \mathcal{Q}_{\nu}^{b}} \\
{\left[\mathcal{R}_{\nu}, \mathcal{S}_{\nu}^{a}\right]=-i \epsilon^{a b} \mathcal{S}_{\nu}^{b},} \\
{\left[\mathcal{G}_{\nu}^{-}, \mathcal{Q}_{\nu}^{a}\right]=2\left(\mathcal{S}_{\nu}^{a}+i \epsilon^{a b} \mathcal{S}_{\nu}^{b}\right)} \\
{\left[\mathcal{G}_{\nu}^{+}, \mathcal{Q}_{\nu}^{a}\right]=-2\left(\mathcal{S}_{\nu}^{a}-i \epsilon^{a b} \mathcal{S}_{\nu}^{b}\right)} \\
{\left[\mathcal{G}_{\nu}^{-}, \mathcal{S}_{\nu}^{a}\right]=2\left(\mathcal{Q}_{\nu}^{a}-i \epsilon^{a b} \mathcal{Q}_{\nu}^{b}\right)} \\
{\left[\mathcal{G}_{\nu}^{+}, \mathcal{S}_{\nu}^{a}\right]=-2\left(\mathcal{Q}_{\nu}^{a}+i \epsilon^{a b} \mathcal{Q}_{\nu}^{b}\right),} \\
\left\{\mathcal{Q}_{\nu}^{a}, \mathcal{Q}_{\nu}^{b}\right\}=2 \delta^{a b} \mathcal{H}_{\nu}^{e}, \\
\left\{\mathcal{S}_{\nu}^{a}, \mathcal{S}_{\nu}^{b}\right\}=2 \delta^{a b}\left(\mathcal{H}_{\nu}^{e}-4 \mathcal{R}_{\nu}\right), \\
\left\{\mathcal{Q}_{\nu}^{a}, \mathcal{S}_{\nu}^{b}\right\}=\delta^{a b}\left(\mathcal{G}_{\nu}^{+}+\mathcal{G}_{\nu}^{-}\right)+i \epsilon^{a b}\left(\mathcal{G}_{\nu}^{+}-\mathcal{G}_{\nu}^{-}\right)
\end{gathered}
$$

correspond to the dynamical $\mathfrak{o} \mathfrak{s} \mathfrak{p}(2,2)$ superconformal symmetry of the system (4.30). To identify the generators of superconformal symmetry of the system (4.31), we note that the superconformal $\mathfrak{o} \mathfrak{s} \mathfrak{p}(2,2)$ algebra given by Lie super-algebraic relations (4.35)(4.41) has an automorphism $f=f^{-1}$ which corresponds to the transformations $\mathcal{H}_{\nu}^{e} \rightarrow \mathcal{H}_{\nu}^{e}-4 \mathcal{R}_{\nu}=\mathcal{H}_{\nu}^{b}, \mathcal{R}_{\nu} \rightarrow-\mathcal{R}_{\nu}$, $\mathcal{G}_{\nu}^{ \pm} \rightarrow \mathcal{G}_{\nu}^{ \pm}, \mathcal{Q}_{\nu}^{1} \rightarrow-\mathcal{S}_{\nu}^{1}, \mathcal{Q}_{\nu}^{2} \rightarrow \mathcal{S}_{\nu}^{2}, \mathcal{S}_{\nu}^{1} \rightarrow-\mathcal{Q}_{\nu}^{1} \mathcal{S}_{\nu}^{2} \rightarrow \mathcal{Q}_{\nu}^{2}$. Therefore, the transformed operators are the generators of the $\mathfrak{o} \mathfrak{g} \mathfrak{p}(2,2)$ corresponding to the system $\mathcal{H}_{\nu}^{b}$, for which the Poincaré supercharges are $-\mathcal{S}_{\nu}^{1}$ and $\mathcal{S}_{\nu}^{2}$. None of these supercharge operators annihilates both ground states of the system coherently with spontaneously broken nature of the $\mathcal{N}=2$ Poincaré supersymmetry of the system (4.31).

Let us consider now the action of the $K_{4}$ group on the superextended systems. The action of both generators $\rho_{1}$ and $\rho_{2}$ on superextended systems we define in the same way as they were defined for the nonextended AFF system $\mathcal{H}_{\nu}$. We use Eq. (4.28) to transform the generators of $\mathfrak{o} \mathfrak{g} \mathfrak{p}(2,2)$ by $\rho_{1}$, and obtain

$$
\begin{gathered}
\rho_{1}\left(\mathcal{H}_{\nu}^{e}\right)=\sigma_{1}\left(\mathcal{H}_{\nu-1}^{e}-4 \mathcal{R}_{\nu-1}\right) \sigma_{1}, \\
\rho_{1}\left(\mathcal{G}_{\nu}^{ \pm}\right)=\sigma_{1}\left(\mathcal{G}_{\nu-1}^{ \pm}\right) \sigma_{1}, \\
\rho_{1}\left(\mathcal{R}_{\nu}\right)=\sigma_{1}\left(-\mathcal{R}_{\nu-1}\right) \sigma_{1}, \\
\rho_{1}\left(\mathcal{Q}_{\nu}^{1}\right)=\sigma_{1}\left(-\mathcal{S}_{\nu-1}^{1}\right) \sigma_{1}, \quad \rho_{1}\left(\mathcal{Q}_{\nu}^{2}\right)=\sigma_{1}\left(\mathcal{S}_{\nu-1}^{2}\right) \sigma_{1},
\end{gathered}
$$

$\rho_{1}\left(\mathcal{S}_{\nu}^{1}\right)=\sigma_{1}\left(-\mathcal{Q}_{\nu-1}^{1}\right) \sigma_{1}, \quad \rho_{1}\left(\mathcal{S}_{\nu}^{2}\right)=\sigma_{1}\left(\mathcal{Q}_{\nu-1}^{2}\right) \sigma_{1}$. 
Therefore, the action of $\rho_{1}$ on generators of the superconformal algebra of the system described by the superHamiltonian $\mathcal{H}_{\nu}^{e}$ produces the generators of supeconformal symmetry $\mathfrak{o} \mathfrak{g} \mathfrak{p}(2,2)$ of the system described by the superHamiltonian $\mathcal{H}_{\nu-1}^{b}$ unitarily rotated by $\sigma_{1}$. In other words, the action of $\rho_{1}$ on the system (4.30) and generators of its superconformal algebra is equivalent to do the shift $\nu \rightarrow \nu-1$, apply the automorphism $f$ defined above, and then apply a unitary transformation given by the operator $\sigma_{1}$. The transformed generators (4.42)-(4.45) still satisfy the same superconformal algebra, i.e., $\rho_{1}$ is an automorphism of the $\mathfrak{o} \mathfrak{g} \mathfrak{p}(2,2)$, but they describe another superextended system having different properties in the sense that in the transformed system, unlike the initial system (4.30), the $\mathcal{N}=2$ Poincaré supersymmetry is spontaneously broken in the case of $\nu>-1 / 2$. The only exception from this rule corresponds to the case $\nu=-1 / 2$, where the transformed Hamiltonian reduces to $\sigma_{1} \mathcal{H}_{-1 / 2}^{e} \sigma_{1}$, and represents a unitary transformed super-Hamiltonian with the unbroken $\mathcal{N}=2$ Poincaré supersymmetry.

On the other hand, one can verify that when $\rho_{1}$ acts on the Hamiltonian $\mathcal{H}_{\nu}^{b}$, it produces $\sigma_{1}\left(\mathcal{H}_{\nu-1}^{e}\right) \sigma_{1}$, and this time the $\mathcal{N}=2$ Poincare supersymmetry of the system is changed from the spontaneously broken phase (in the case of $\nu>-1 / 2$ ) to the phase of unbroken supersymmetry, with the only exception of the system $\mathcal{H}_{-1 / 2}^{b}$ with unbroken supersymmetry, which unitary transforms into $\sigma_{1} \mathcal{H}_{-1 / 2}^{b} \sigma_{1}$. This picture of transformation of $\rho_{1}$ on superextended systems can be compared with the case of the nonextended AFF system, where $\rho_{1}$ acts identically on its Hamiltonian and generators of the conformal symmetry, though, as we saw, it acts nontrivially on eigenstates of the system.

By knowing the action of $\rho_{2}$ on the intertwining operators (4.25), explicitly given in (4.29), we can apply this transformation to the generators of superconformal algebra. As a result, we find that it generates the automorphism of $\mathfrak{o} \mathfrak{s} \mathfrak{p}(2,2)$ given by relations

$$
\begin{array}{r}
\rho_{2}\left(\mathcal{H}_{\nu}^{e}\right)=-\mathcal{H}_{\nu}^{b}, \quad \rho_{2}\left(\mathcal{G}_{\nu}^{ \pm}\right)=-\mathcal{G}_{\nu}^{\mp}, \quad \rho_{2}\left(\mathcal{R}_{\nu}\right)=\mathcal{R}_{\nu}, \\
\rho_{2}\left(\mathcal{Q}_{\nu}^{1}\right)=-i \mathcal{S}_{\nu}^{1}, \quad \rho_{2}\left(\mathcal{Q}_{\nu}^{2}\right)=-i \mathcal{S}_{\nu}^{2}, \\
\rho_{2}\left(\mathcal{S}_{\nu}^{1}\right)=-i \mathcal{Q}_{\nu}^{1}, \quad \rho_{2}\left(\mathcal{S}_{\nu}^{2}\right)=-i \mathcal{Q}_{\nu}^{2} .
\end{array}
$$

The transformed Hamiltonian operator is similar here to the Hamiltonian produced by the automorphism $f$ but multiplied by -1 . This correlates with the anti-Hermitian nature of the transformed fermion generators of superalgebra. Accordingly, the spectrum of the transformed matrix Hamiltonian is negative, not bounded from below, and each its level is doubly degenerate for $\nu \geq-1 / 2$.

In correspondence with the described picture, the application of the combined transformation $\rho_{2} \rho_{1}$ is just another automorphism of the superconformal algebra (4.35)(4.41), which produces anti-Hermitian odd generators, and $\rho_{2} \rho_{1}\left(\mathcal{H}_{\nu}^{e}\right)=\sigma_{1}\left(-\mathcal{H}_{\nu-1}^{e}\right) \sigma_{1}$. The discrete spectrum of the transformed Hamiltonian is not restricted from below and is given by the numbers $\mathcal{E}_{n}=-4 n, n=0,1, \ldots$, where each negative energy level is doubly degenerate, while nondegenerate zero energy level corresponds to the state $\left(\psi_{\nu, 0}, 0\right)^{t}$.

\section{DUAL DARBOUX SCHEMES}

If we have two ways to generate the same, modulo an additive shift, particular system from the AFF model with a certain value of the parameter $\nu$ by employing two distinct Darboux transformations based on distinct sets of the seed states with different behavior at infinity, we say that we have two dual Darboux schemes, or a Darboux duality. This property was discussed and exploited earlier in the case of rational deformations of harmonic oscillator for the construction of the complete sets of the spectrum generating ladder operators as well as for the description of emergent nonlinear extensions of superconformal symmetry appearing in such systems $[42,43,48,51,52]$. We also used dual Darboux schemes in the previous section in the discussion of supersymmetric extensions of the AFF model.

To give a simple example of the dual schemes, one can choose the set of eigenstates $\left(\psi_{\nu, 0}, \ldots, \psi_{\nu, m-1}\right)$, whose Wronskian is, up to a multiplicative constant, $x^{m(2 \nu+m+1) / 2} e^{-m x^{2} / 2}$. This implies that after the Darboux transformation (2.2) the Hamiltonian operator takes the form $\mathcal{H}_{\nu+m}+2 m$. On the other hand, if we take the scheme $\left(\psi_{\nu,-0}, \ldots, \psi_{\nu,-(m-1)}\right)$ based on nonphysical eigenstates generated by transformation $\rho_{2}$, we produce the same system but shifted by the relative constant $-4 m$, so this both schemes are dual. Intertwining operators of order $m$ of both schemes allow to construct fermionic integrals of motion of the corresponding supersymmetric extension of the system, and generate the corresponding $\mathfrak{o} \mathfrak{s} \mathfrak{p}(2,2)$ superconformal symmetry in the case of $m=1$, as it was done in the previous section, or to generate nonlinearly deformed and extended version of $\mathfrak{o} \mathfrak{g} \mathfrak{p}(2,2)$ when $m>1$.

The purpose of this section is to derive certain Wronskian identities and employ them for construction of the dual Darboux schemes. The latter allow us to generate rational deformations of a general form with arbitrary number of gaps implemented into equidistant spectrum of the AFF systems in a well controlled manner, to identify the complete sets of the spectrum generating ladder operators for them and their nonlinearly deformed conformal symmetries. We also observe the peculiar changes that happen with spectra of such systems under variation of the parameter $\nu$ when it goes through half-integer values.

In the following the equalities between wave functions and Wronskians are considered up to multiplication by nonzero real constants. 


\section{A. The dual schemes algorithm}

Here we consider a systematic procedure to obtain the dual scheme from a given Darboux scheme by means of certain Wronskian identities.

As we have shown in the previous section, the case in which $\nu$ takes half-integer values is special and more complicated in comparison with the nonhalf-integer case. This happens due to appearance of Jordan states in the game via the properties of noncompact generators of the conformal symmetry which simultaneously are the ladder operators for corresponding AFF systems, see Eq. (4.23). By this reason we start first with the case of nonhalf-integer values of $\nu$, and choose a generic set of physical and nonphysical eigenstates of $\mathcal{H}_{\nu}$ as the seed states,

$$
\begin{aligned}
\{\alpha\} & =\left(\psi_{\nu, k_{1}}, \ldots, \psi_{\nu, k_{N_{1}}}, \psi_{-\nu-1, l_{1}}, \ldots, \psi_{-\nu-1, l_{N_{2}}}\right), \\
k_{i}, l_{j} & = \pm 0, \pm 1, \ldots,
\end{aligned}
$$

where $i=1, \ldots, N_{1}$ and $j=1, \ldots, N_{2}$, and, for simplicity, we suppose that $\left|k_{1}\right|<\ldots<\left|k_{N_{1}}\right|$ and $\left|l_{1}\right|<\ldots<\left|l_{N_{2}}\right|$.

Consider now a scheme of the form (5.1) with nonrepeated states, and suppose that both $k_{i}$ and $l_{j}$ carry the same sign for all $i$ and $j$. Also let us define the index number $n_{N}=\max \left(\left|k_{1}\right|, \ldots,\left|k_{N_{1}}\right|,\left|l_{1}\right|, \ldots,\left|l_{N_{2}}\right|\right)$, which can correspond to a state with index $\nu$ or $-\nu-1$. So if $k_{i}$ and $l_{j}$ carry the plus sign, the equality

$$
\begin{aligned}
W(\{\alpha\})= & e^{-\left(n_{N}+1\right) x^{2}} W\left(\left\{\Delta_{-}\right\}\right), \\
\left\{\Delta_{-}\right\}:= & \left(\psi_{-\nu-1,-0}, \psi_{\nu,-0}, \ldots, \check{\psi}_{-\nu-1,-r_{i}}, \check{\psi}_{\nu,-s_{i}}, \ldots,\right. \\
& \left.\psi_{-\nu-1,-n_{N}}, \psi_{\nu,-n_{N}}\right),
\end{aligned}
$$

is satisfied, where the marked states $\check{\psi}_{-\nu-1,-r_{i}}$ and $\check{\psi}_{\nu,-s_{i}}$, with $r_{i}=n_{N}-k_{i}$ and $s_{j}=n_{N}-l_{j}$, are omitted from the set $\left\{\Delta_{-}\right\}$. On the contrary, if $k_{i}$ and $l_{j}$ carry the minus sign, we have the equality

$$
\begin{aligned}
W(\{\alpha\})= & e^{\left(n_{N}+1\right) x^{2}} W\left(\left\{\Delta_{+}\right\}\right), \\
\left\{\Delta_{+}\right\}:= & \left(\psi_{-\nu-1,0}, \psi_{\nu, 0}, \ldots, \check{\psi}_{-\nu-1, r_{i}}, \check{\psi}_{\nu, s_{j}}, \ldots,\right. \\
& \left.\psi_{-\nu-1, n_{N}}, \psi_{\nu, n_{N}}\right),
\end{aligned}
$$

where now $r_{i}=n_{N}-\left|k_{i}\right|$ and $s_{j}=n_{N}-\left|l_{j}\right|$. These relations are also valid if one of the numbers $N_{1}$ or $N_{2}$ is equal to zero, which means that in the corresponding scheme there are only states of the same kind with respect to the first index, $-\nu-1$ or $\nu$, respectively.

When considering $\nu=\ell-1 / 2$ with $\ell=0,1$, 2, we will have relations analogous to (5.2) and (5.3), but changing each state of the form $\psi_{-\nu-1, \pm(\ell+k)}$ by $\Omega_{\ell-1 / 2, \pm k}$, as a result we deal with the confluent Darboux transformation.

To prove the displayed Wronskian relations, we have to keep in mind that the operators $\mathcal{C}_{\nu}^{ \pm}$are in fact the intertwining operators of the dual schemes $\left(\psi_{\nu, 0}, \psi_{-\nu-1,0}\right)$ and $\left(\psi_{\nu,-0}, \psi_{-\nu-1,-0}\right)$ in the case of $\nu>1 / 2$, while for $\nu=-1 / 2$ we take the sets $\left(\psi_{1 / 2,0}, \Omega_{-1 / 2,0}\right)$ and $\left(\psi_{1 / 2,-0}, \Omega_{-1 / 2,-0}\right)$. It is also necessary to take into account the action of these operators on eigenstates and Jordan states discussed in the previous section.

By means of Eqs. (2.8), (2.9), (4.19) and (4.21), we will develop a step by step processes to show the validity of (5.2) and (5.3) in the case when $\nu$ is not a half-integer number, and then we will explain how these relations can be extended to the half-integer case. As a starting point, consider the Wronskian of the set $\{\alpha\}$ defined in (5.1). If the states $\psi_{\nu, \pm 0}$ and $\psi_{-\nu-1, \pm 0}$ do not belong to (5.1), we can replace the Wronskian $W(\{\alpha\})$ by

$$
\begin{gathered}
W\left(\psi_{\nu, \pm 0}, \psi_{-\nu-1, \pm 0}, \tilde{\psi}_{\nu, \pm 0}, \tilde{\psi}_{-\nu-1, \pm 0},\{\alpha\}\right) \\
=e^{\mp x^{2}} W\left(\psi_{\nu, \mp 0}, \psi_{-\nu-1, \mp 0},\left\{\mathcal{C}_{\nu}^{\mp} \alpha\right\}\right),
\end{gathered}
$$

where we used relations (2.8), (2.9), (4.19) and (4.20), and $\left\{\mathcal{C}_{\nu}^{\mp} \alpha\right\}$ means that the ladder operators are applied to all the states in the set. On the other hand, if $\psi_{r(\nu), \pm 0}$ belong to (5.1), we can replace the Wronskian of the initial set of the seed states by

$$
\begin{aligned}
& W\left(\psi_{r(-\nu-1), \pm 0}, \tilde{\psi}_{r(-\nu-1), \pm 0},\{\alpha\}\right) \\
& \quad=e^{\mp x^{2}} W\left(\psi_{r(-\nu-1), \mp 0},\left\{\mathcal{C}_{\nu}^{\mp} \beta_{1}\right\}\right),
\end{aligned}
$$

where $\left\{\beta_{1}\right\}$ is the scheme $\{\alpha\}$ with the omitted state $\psi_{r(\nu), \pm 0}$. Finally, if $\psi_{\nu, \pm 0}$ and $\psi_{-\nu-1, \pm 0}$ belong to (5.1), we have

$$
W(\{\alpha\})=e^{\mp x^{2}} W\left(\left\{\mathcal{C}_{\nu}^{\mp} \beta_{2}\right\}\right),
$$

where $\left\{\beta_{2}\right\}$ is the scheme $\{\alpha\}$ with the omitted states $\psi_{\nu, \pm 0}$ and $\psi_{-\nu-1, \pm 0}$. Note that in all these three relations we have lowered or raised the index of the states in $\{\alpha\}$, and also in the case of Eqs. (5.4) and (5.5) we have included additional states which do not belong to the initial set. Also, we note that an exponential factor has appeared. These identities can be applied to the Wronskians on the right-hand side of Eqs. (5.4)-(5.6), which will contribute with new exponential factors in new Wronskians, and so on. For this reason, if we restrict the initial set $\{\alpha\}$ by the conditions described above (that every state in the set has the second index of the same sign), and we repeat this procedure $n_{N}+1$ times with positive (negative) sign of the indexes in (5.4)-(5.6), we finally obtain Eq. (5.2) or (5.3).

Now, we consider the case when $\nu$ takes a half-integer value. Note first that by means of relations (2.8), (2.19), the second relation in (4.19), and relations (4.21), we can repeat the arguments presented above for the case when $\nu=-1 / 2$, but changing each function of the form $\psi_{-\nu-1, n}$ by $\Omega_{-1 / 2, n}$ in relations (5.4)-(5.6). As a consequence, relations (5.2) and (5.3) are now valid with the same corresponding changes. On the other hand, with a simple 
(a)

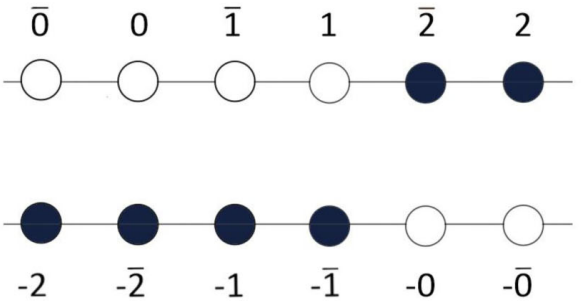

(b)

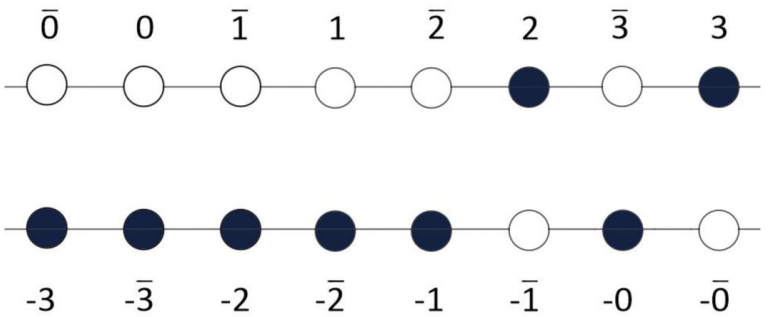

FIG. 2. Two "mirror diagrams" corresponding to dual schemes for the conformal mechanics model. The numbers $n$ indicate the states $\psi_{\nu, n}$, and symbols $\bar{n}$ correspond to the states $\psi_{-\nu-1, n}$.

example one can see that this does not hold for the case $\nu=\ell-1 / 2$ with $\ell \geq 1$. For this we consider the scheme $\left(\psi_{1 / 2,1}, \psi_{1 / 2,2}\right)$, for which the Wronskian can be rewritten as

$$
\begin{gathered}
W\left(\psi_{1 / 2,0}, \psi_{3 / 2,0}, \tilde{\psi}_{1 / 2,0}, \tilde{\psi}_{-3 / 2,0}, \psi_{1 / 2,1}, \psi_{1 / 2,2}\right) \\
=e^{-x^{2}} W\left(\tilde{\psi}_{-3 / 2,0}, \psi_{1 / 2,-0}, \psi_{1 / 2,0}, \psi_{1 / 2,1}\right),
\end{gathered}
$$

where we have repeated the same idea that we employed in (5.4), and also we used (4.9) to change $\psi_{-3 / 2,-0}$ by $\tilde{\psi}_{-3 / 2,0}$. As this last indicated state appears, we cannot use Eqs. (2.8) and (2.9) to include $\psi_{-3 / 2,0}$ and produce the intertwining operator $\mathcal{C}_{1 / 2}^{-}$, so the algorithm is stopped.

Nevertheless, we can use the connection between $\mathcal{H}_{-1 / 2+\ell}$ and $\mathcal{H}_{-1 / 2}$, provided by the Darboux transformation produced by the seed states $\left(\psi_{-1 / 2, \pm 0}, \ldots, \psi_{-1 / 2, \pm(\ell-1)}\right)$ to obtain the corresponding dual schemes. Each eigenstate or Jordan state of $\mathcal{H}_{\nu+m}$ can be obtained by applying the Darboux mapping to corresponding eigenstates or Jordan states of $\mathcal{H}_{-1 / 2}$. The details of the procedure are described in the Appendix A, but it can be summarized in three simple steps.

(i) If we have a scheme based on eigenstates or Jordan states of $\mathcal{H}_{\nu+m}$ with $\nu=\ell-1 / 2$, then by using the corresponding Darboux transformation and Eq. (2.8), we find an equivalent scheme in the system with $\nu=-1 / 2$.

(ii) Then we construct the dual scheme by using the algorithm adapted for $\nu=-1 / 2$.

(iii) Finally, we translate the resulting dual scheme into the scheme for $H_{\nu+m}$ by using the corresponding Darboux transformation and Eq. (2.8) again.

The main result is that we just have to change every function of the form $\psi_{-\nu-1, \pm(\ell+n)}$ by $\Omega_{-\ell-1 / 2, \pm n}$ when $\nu$ is equal to $\ell-1 / 2$. In this way one finds that $W\left(\psi_{1 / 2,1}\right.$, $\left.\psi_{1 / 2,2}\right)=e^{-3 x^{2}} W\left(\psi_{1 / 2,-0}, \psi_{1 / 2,-1}, \psi_{1 / 2,-2}, \Omega_{1 / 2,-1}\right)$.

Now, we focus our discussion on relation between both dual schemes. In general, if $\left\{\Delta_{-}\right\}$coincides with the scheme in the argument of the Wronskian on the left-hand side of (5.3), then $\left\{\Delta_{+}\right\}$coincides with the scheme on the left-hand side of (5.2), and consequently $W\left(\left\{\Delta_{+}\right\}\right)=$ $e^{-\left(n_{N}+1\right) x^{2}} W\left(\left\{\Delta_{-}\right\}\right)$. By this reason we call $\left\{\Delta_{+}\right\}$and $\left\{\Delta_{-}\right\}$a positive and a negative dual scheme, respectively.
If $\left\{\Delta_{+}\right\}$has $n_{+}$states and $\left\{\Delta_{-}\right\}$has $n_{-}$states, then one can note that $n_{N}=n_{n_{+}}=n_{n_{-}}$, and $n_{+}+n_{-}=2\left(n_{N}+1\right)$, which is the total number of the states employed in both dual transformations.

The general rules can be summarized and better understood with the examples presented diagrammatically in Fig. 2.

This kind of diagrams is read as follows. In the top-line, there appear the ordered states vanishing at infinity, which are ordered from the lowest to the highest second index in wave functions, and which always end in the number without a bar (the first index of wave function is $\nu$ ). In the bottom-line, there appear the Wick rotated states (second index of wave functions appears with the minus sign), ordered in the same way. The filled black circles denote the states that appear in the Wronskian arguments in the corresponding dual scheme. The mirror diagrams shown in Fig. 2 correspond to the following Wronskian equalities:

$W\left(\psi_{-\nu-1,2}, \psi_{\nu, 2}\right)=e^{-3 x^{2}} W\left(\psi_{-\nu-1,-1}, \psi_{\nu,-1}, \psi_{-\nu-1,-2}, \psi_{\nu,-2}\right)$,

$$
\begin{aligned}
W\left(\psi_{\nu, 2}, \psi_{\nu, 3}\right)= & e^{-4 x^{2}} W\left(\psi_{\nu,-0}, \psi_{\nu,-1}, \psi_{-\nu-1,-2}, \psi_{\nu,-2},\right. \\
& \left.\psi_{-\nu-1,-3}, \psi_{\nu,-3}\right),
\end{aligned}
$$

whose explicit form is given in Appendix C. The transformation which relates the AFF systems described by $\mathcal{H}_{\nu}$ with $\mathcal{H}_{\nu+m}$ can also be understood within this picture. Furthermore, using a diagram similar to those in Fig. 2, one can show that the schemes $\left\{\Delta_{+}\right\}=\left(\psi_{r(\nu), 0}, \ldots, \psi_{r(\nu), m-1}\right)$ and $\left\{\Delta_{-}\right\}=\left(\psi_{r(\nu),-0}, \ldots, \psi_{r(\nu),-(m-1)}\right)$ are dual.

\section{RATIONALLY DEFORMED AFF SYSTEMS}

A rational deformation of the AFF model can be generated by taking a set of the seed states

$$
\left\{\alpha_{K A}\right\}=\left(\psi_{\nu, l_{1}}, \psi_{\nu, l_{1}+1}, \ldots, \psi_{\nu, l_{m}}, \psi_{\nu, l_{m}+1}\right),
$$

composed from $m$ pairs of neighbor physical states. KreinAdler theorem $[37,38]$ guarantees that the resulting system described by the Hamiltonian operator of the form 


$$
\mathcal{H}_{(\nu, m)}^{K A}=\mathcal{H}_{\nu+m}+4 m+\frac{F_{\nu}(x)}{Q_{\nu}(x)}
$$

is nonsingular on $\mathbb{R}^{+}$. Here $F_{\nu}(x)$ and $Q_{\nu}(x)$ are real-valued polynomials, $Q_{\nu}(x)$ has no zeroes on $\mathbb{R}^{+}$, its degree is two more than that of $F_{\nu}(x)$, and so, the last rational term in (6.2) vanishes at infinity. The spectrum of the system (6.2) is the equidistant spectrum of the AFF model with the removed energy levels corresponding to the seed states. Consequently, any gap in the resulting system has a size $12+8 k$, where $k=0,1, \ldots$ corresponds to $k$ adjacent pairs in the set (6.1) which produce a given gap. An example of this kind of the systems is generated by the scheme $\left(\psi_{\nu, 2}, \psi_{\nu, 3}\right)$, whose dual negative scheme is given by Eq. (5.9).

Another class of rationally extended AFF systems is provided by isospectral deformations generated by the schemes of the form

$$
\left\{\alpha_{\mathrm{iso}}\right\}=\left(\psi_{\nu,-s_{1}}, \ldots, \psi_{\nu,-s_{m}}\right)
$$

which contain only Wick rotated states $\rho_{2}\left(\psi_{\nu, n}(x)\right)=$ $\psi_{\nu, n}(i x)$. As the functions used in this scheme are proportional to $x^{\nu+1}$ and do not have real zeros other than $x=0$, one obtains a regular on $\mathbb{R}^{+}$system of the form

$$
\mathcal{H}_{(\nu, m)}^{\text {iso }}=\mathcal{H}_{\nu+m}+2 m+f_{\nu}(x)
$$

where $f_{\nu}(x)$ is a rational function disappearing at infinity [64], and one can find that potential of the system (6.4) is a convex on $\mathbb{R}^{+}$function. In this case the transformation does not remove or add energy levels, and, consequently, the initial system $\mathcal{H}_{\nu}$ and the deformed system (6.4) are completely isospectral superpartners. Some concrete examples of the systems (6.4) with integer values of $\nu$ were considered in [42].

Consider yet another generalized Darboux scheme which allows us to interpolate between different rationally deformed AFF systems. For this we assume that the initial AFF system is characterized by the parameter $\nu=\mu+m$, where $-1 / 2<\mu \leq 1 / 2$ and $m$ can take any non-negative integer value. For these ranges of values of the parameter $\nu$, real zeros of the functions $\psi_{\mu+m, n-m}$ are located between zeros of $\psi_{-(\mu+m)-1, n}$, so that we can rethink the Krein-Adler theorem and consider the scheme

$$
\begin{aligned}
\left\{\gamma_{\mu}\right\}= & \left(\psi_{-(\mu+m)-1, n_{1}}, \psi_{(\mu+m), n_{1}-m}, \ldots, \psi_{-(\mu+m)-1, n_{N}},\right. \\
& \left.\psi_{(\mu+m), n_{N}-m}\right),
\end{aligned}
$$

which includes $2 N$ states and where we suppose that $n_{i}-$ $m \geq 0$ for all $i=1, \ldots, N$. The Darboux transformation based on the set (6.5) produces the system

$$
\begin{aligned}
\mathcal{H}_{\mu+m}^{\text {def }} & :=\mathcal{H}_{\mu+m}-2\left(\ln W\left(\gamma_{\nu}\right)\right)^{\prime \prime} \\
& =\mathcal{H}_{\mu+m}+4 N+h_{\mu+m}(x) / q_{\mu+m}(x),
\end{aligned}
$$

where the term $4 N$ is provided by the Gaussian factor in the Wronskian, and the last term is a rational function vanishing at infinity and having no zeros in the whole real line, including the origin, if an only if $-1 / 2<\mu \leq 1 / 2$, see Appendix B. Let us analyze now some special values of $\mu$.

The case $\mu=0$ : by virtue of relation between Laguerre and Hermite polynomials, $H_{2 n}(x)=(-4)^{n} n ! L_{n}^{(-1 / 2)}\left(x^{2}\right)$ and $H_{2 n+1}(x)=2(-4)^{n} n ! x L_{n}^{(1 / 2)}\left(x^{2}\right)$, in this case we obtain those systems which were generated in [42] by Darboux transformations of the half-harmonic oscillator. They are characterized by gaps of the size $8+4 k$, and represent rational extensions of the AFF model with integer coupling constant $g=m(m+1)$, which in the case of $m=0$ reduce to a rationally extended harmonic oscillator supplied with a potential barrier at $x=0$. Note that the minimal size of the gaps here is less than that for the systems produced by the scheme (6.1).

The case $\mu=1 / 2$ : we have here the relation $\rho_{1}\left(\psi_{m+1 / 2, n_{i}}\right)=\psi_{-m-3 / 2, n_{i}}=(-1)^{m+1} \psi_{m+1 / 2, n_{i}-m-1}$, due to which the scheme (6.5) transforms into

$$
\begin{gathered}
\left\{\gamma_{1 / 2}\right\}=\left(\psi_{1 / 2+m, n_{1}-m-1}, \psi_{1 / 2+m, n_{1}-m}, \ldots,\right. \\
\left.\psi_{1 / 2+m, n_{N}-m-1}, \psi_{1 / 2+m, n_{N}-m}\right),
\end{gathered}
$$

which corresponds to (6.1) with $l_{i}=n_{i}-m-1$. We additionally suppose that $n_{i}-m-1 \neq n_{i-1}-m$, otherwise the Wronskian vanishes. Note that when $\mu \neq 1 / 2$, the image of the states $\psi_{\mu+m, n_{i}-m-1}$ under Darboux mapping (2.3) is a physical state, but in the case $\mu=1 / 2$ such states are mapped into zero since the argument $\psi_{1 / 2+m, n_{i}-m-1}$ appears twice in the Wronskian of the numerator.

The case $\mu=-1 / 2$ : this case was not included in the range of $\mu$ from the beginning due to relation $\rho_{1}\left(\psi_{m-1 / 2, n_{i}}\right)=$ $\psi_{-m-1 / 2, n_{i}}=(-1)^{m} \psi_{m-1 / 2, n_{i}-m}$ which would mean the appearance of the repeated states in the scheme (6.5) and vanishing of the corresponding Wronskian. However, in Appendix B we show that the limit relation $\lim _{\mu \rightarrow-1 / 2} W$ $\left(\left\{\gamma_{\mu}\right\}\right) /\left(\mu+\frac{1}{2}\right)^{N} \propto W(\{\gamma\})$ is valid, where the scheme $\{\gamma\}$ is

$$
\begin{aligned}
\{\gamma\}= & \left(\psi_{m-1 / 2, n_{1}-m}, \Omega_{m-1 / 2, n_{1}-m}, \ldots, \psi_{m-1 / 2, n_{N}-m},\right. \\
& \left.\Omega_{m-1 / 2, n_{N}-m}\right),
\end{aligned}
$$

which corresponds to a non-singular confluent Darboux transformation [45].

By considering this last comment, in conclusion we have that when $-1 / 2 \leq \mu<1 / 2$, the states $\psi_{-(\mu+m)-1, n_{i}}$ (and $\Omega_{m-1 / 2, n_{i}-m}$ in the case of $\mu=-1 / 2$ ) are nonphysical states. This means that only the physical states $\psi_{\nu+m, n_{i}-m}$ indicate the energy levels removed under the corresponding 

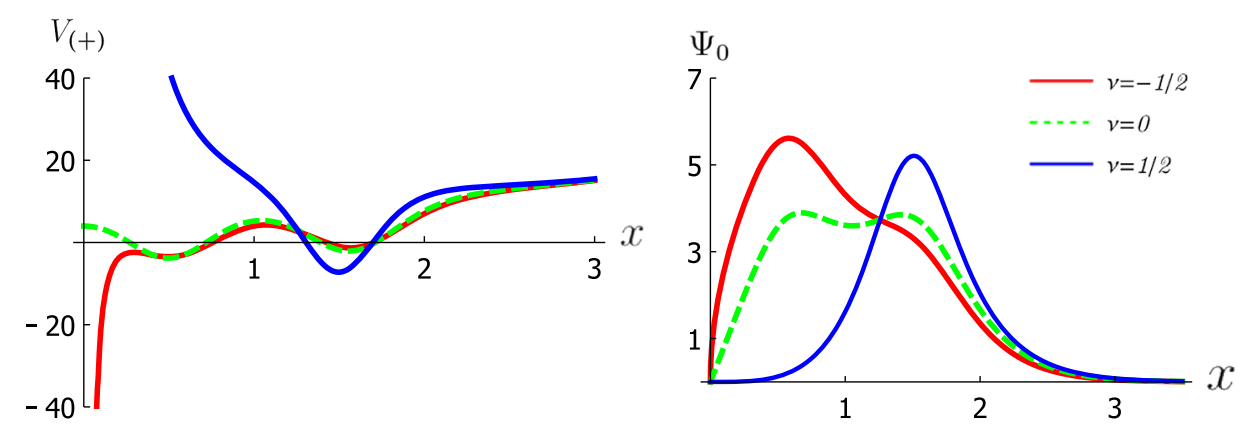

FIG. 3. On the left, a graph of the corresponding potential is shown which is produced by the associated Darboux transformation applied to the AFF model with three indicated values of the parameter $\nu$ versus the dimensionless coordinate $x$. For $\nu=-1 / 2$, the corresponding limit is taken, and the resulting system has an attractive potential with a (not shown) potential barrier at $x=0$. For $\nu=0$, we obtain a rationally extended half-harmonic oscillator. The case $\nu=1 / 2$ corresponds to the Krein-Adler scheme $\left(\psi_{1 / 2,1}, \psi_{1 / 2,2}\right)$ with a gap equal to 12. On the right, the ground states of the corresponding generated systems are shown as functions of dimensionless coordinate $x$.
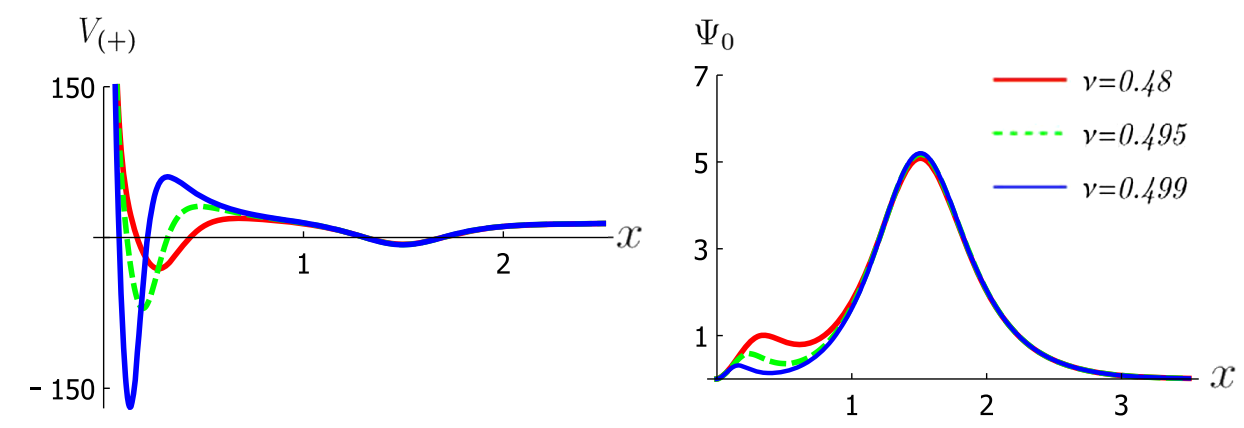

FIG. 4. On the left, the potential of deformed systems with $\nu$ close to $1 / 2$ is shown. On the right, the ground states of the corresponding systems are displayed.

Darboux transformation, i.e., there are gaps of the minimum size $2 \Delta E=8$, where $\Delta E=4$ is the distance between energy levels of the AFF model, which can merge to produce energy gaps of the size $8+4 k$. On the other hand, when $\mu=1 / 2$, we have a typical Krein-Adler scheme with gaps of the size $12+4 k$.

To give an example, we put $m=0$, that means $\nu=\mu$, and consider the scheme $\left(\psi_{-\nu-1,2}, \psi_{\nu, 2}\right)$ with $-1 / 2<$ $\nu \leq 1 / 2$, whose Wronskian is presented explicitly in Appendix $\mathrm{C}$, and in the case of $\nu=-1 / 2$ we have the scheme $\left(\psi_{-1 / 2,2}, \Omega_{-1 / 2,2}\right)$. The potential of the rationally deformed AFF system generated by the corresponding Darboux transformation is shown in Figs. 3 and 4.

As it is seen from the figures, the first minimum of the potential grows in its absolute value, its position moves to 0 , and it disappears at $\nu=1 / 2$, while the local maximum near zero also grows, its position approaches zero, and it goes to infinity in the limit. Besides, the first maximum of the ground state vanishes when $\nu$ approximates the limit value $1 / 2$. Coherently with the described behavior of the potential, the image of the Darboux-transformed state $\psi_{\nu, 1}$, which is the first excited state of the new system when $-1 / 2 \leq \nu<1 / 2$, vanishes when $\nu \rightarrow 1 / 2$, the corresponding energy level disappears from the spectrum at $\nu=1 / 2$, and the size of the gap increases from 8 to 12 .

The described three possible selection rules to choose the seed states correspond to the negative scheme (6.3), which generates isospectral deformations, the positive KreinAdler scheme (6.1), and the positive interpolating scheme (6.5). Then we can apply the algorithm constructed in Sec. VA to obtain the corresponding dual schemes for them. The positive and negative dual schemes will be used in the next subsection to construct complete sets of the spectrum generating ladder operators for the rationally deformed conformal mechanics systems.

\section{INTERTWINING AND LADDER OPERATORS}

As a starting point, we consider any positive scheme for the AFF model $\mathcal{H}_{\nu}$ that produces its certain nonsingular rational deformation. For simplicity we do not touch here the schemes that contain Jordan states. However, we have relations (2.18) and (4.24), and relations (5.2) and (5.3) which were extended to such cases with the corresponding substitutions; see the comments for Eq. (A6). This means 
that the properties summarized below are also valid for the schemes containing Jordan states.

Let a positive scheme contains $n_{+}$states labeled by $n_{i}$, $i=1, \ldots, n_{+}$, with $n_{n_{+}}$being the biggest quantum number in the set. We denote by $\mathcal{H}_{(+)}$the system generated by the Darboux transformation based on the set of the chosen seed states. By applying the algorithm from Sec. VA, we obtain the corresponding dual negative scheme with $n_{-}=2 n_{n_{+}}+$ $2-n_{+}$seed states labeled by index $-l_{j}$ with $j=1, \ldots, n_{-}$ and $-l_{n_{-}}=-n_{n_{+}}$. The resulting system of the Darboux transformation based on the negative scheme we denote by $\mathcal{H}_{(-)}$. By using (5.2) we obtain that the generated Schrödinger operators are mutually shifted for a constant,

$$
\begin{aligned}
\mathcal{H}_{(+)}-\mathcal{H}_{(-)} & =\Delta E\left(n_{n_{+}}+1\right)=2\left(n_{+}+n_{-}\right), \\
\Delta E & =4 .
\end{aligned}
$$

We can construct the corresponding intertwining operators of both schemes by following the rule (2.4). Let us denote by $A_{(+)}^{ \pm}$and $A_{(-)}^{ \pm}$the intertwining operators of the positive and negative schemes being differential operators of the orders $n_{+}$and $n_{-}$, respectively. Some useful properties of these operators are summarized as follows. First, they satisfy the intertwining relations

$$
A_{( \pm)}^{-} \mathcal{H}_{\nu}=\mathcal{H}_{( \pm)} A_{( \pm)}^{-}, \quad A_{( \pm)}^{+} \mathcal{H}_{( \pm)}=\mathcal{H}_{\nu} A_{( \pm)}^{+},
$$

from where one concludes that the operators $A_{( \pm)}^{-}$map differently physical eigenstates of $\mathcal{H}_{\nu}$ as well as nonphysical ones obtained from them by action of generators of the $K_{4}$ group. The states $\tilde{\psi}_{r(\nu), \pm n}$ behave asymptotically as $e^{ \pm x^{2} / 2}$, and the states produced from them by application of differential operators $A_{( \pm)}^{-}$will carry the same exponential factor. Having this asymptotic behavior in mind, let us suppose that $\psi_{r(\nu),-l_{*}}$ and $\psi_{r(\nu), n_{*}}$ are some arbitrary states from the negative and positive scheme, respectively. By using (7.2), we obtain the relations

$$
\begin{aligned}
A_{(-)}^{-} \tilde{\psi}_{r(\nu),-l_{*}} & =A_{(+)}^{-} \rho_{1}\left(\psi_{r(\nu), n_{n+}-l_{*}}\right), \\
A_{(+)}^{-} \tilde{\psi}_{r(\nu), n_{*}} & =A_{(-)}^{-} \rho_{1}\left(\psi_{r(\nu),-\left(n_{n+}-n_{*}\right)}\right),
\end{aligned}
$$

in both sides of which the functions satisfy the same second order differential equation and have the same behavior at infinity. Note that in the dual schemes in (5.2) and (5.3), the indexes $n_{n_{+}}-l_{*}$ and $-\left(n_{n_{+}}-n_{*}\right)$ are in correspondence with the indexes $r_{i}$, and $s_{i}$ of the states omitted from the positive and negative scheme, respectively. This helps us to obtain the identities

$$
\begin{aligned}
& A_{(-)}^{+} A_{(+)}^{-}=(-1)^{n_{n_{+}}+1-n_{+}}\left(\mathcal{C}_{\nu}^{-}\right)^{n_{n_{+}}+1}, \\
& A_{(+)}^{+} A_{(-)}^{-}=(-1)^{n_{n_{+}}+1-n_{+}}\left(\mathcal{C}_{\nu}^{+}\right)^{n_{n_{+}}+1}
\end{aligned}
$$

It is enough to prove the first relation in (7.4), and the second is produced by the Hermitian conjugation. As we know, $A_{(+)}^{-}$, annihilates all the states in the positive scheme, while $A_{(-)}^{+}$ annihilates all the functions of the form $A_{(+)}^{-} \tilde{\psi}_{r(\nu),-l_{*}}$. Then, acting by $A_{(-)}^{+}$from the left on both sides of the first relation in (7.3), we find that $\operatorname{ker}\left(A_{(-)}^{+} A_{(+)}^{-}\right)=\left(\psi_{\nu, 0}, \psi_{-\nu-1,0}, \ldots\right.$, $\left.\psi_{\nu, n_{n_{+}}}, \psi_{-\nu-1, n_{n_{+}}}\right)=\operatorname{ker}\left(\mathcal{C}_{\nu}^{-}\right)^{n_{n_{+}}+1}$.

Finally, to have a complete picture we write the relations

$$
\begin{aligned}
A_{(-)}^{-} \psi_{r(\nu), k} & =A_{(+)}^{-} \psi_{r(\nu), n_{n+}+1+k^{\prime}}, \\
A_{(+)}^{-} \psi_{r(\nu),-k^{\prime}} & =A_{(-)}^{-} \psi_{r(\nu),-\left(n_{n+}+1+k^{\prime}\right)} .
\end{aligned}
$$

In the case of the dual schemes $\{\gamma\}$ and $\left\{\Delta_{-}^{\left(m-\frac{1}{2}\right)}\right\}$ defined in (6.8) and (A6), where $\nu=m-1 / 2$, similar relations are obtained but with $\psi_{-\mu-m-1, \pm n_{i}}$ and $\tilde{\psi}_{-\mu-m-1, \pm n_{i}}$ replaced by $\Omega_{m-\frac{1}{2}, \pm\left(n_{i}-m\right)}$ and $\breve{\Omega}_{m-\frac{1}{2}, \pm\left(n_{i}-m\right)}$ when required.

With the help of the described intertwining operators, we can construct three types of ladder operators for $\mathcal{H}_{( \pm)}$ which are given by:

$$
\begin{aligned}
& \mathcal{A}^{ \pm}=A_{(-)}^{-} \mathcal{C}_{\nu}^{ \pm} A_{(-)}^{+}, \quad \mathcal{B}^{ \pm}=A_{(+)}^{-} \mathcal{C}_{\nu}^{ \pm} A_{(+)}^{+}, \\
& \mathcal{C}^{+}=A_{(-)}^{-} A_{(+)}^{+}, \quad \mathcal{C}^{-}=A_{(+)}^{-} A_{(-)}^{+} .
\end{aligned}
$$

Let us denote these operators in the compact form $\mathcal{F}_{a}^{ \pm}=$ $\left(\mathcal{A}^{ \pm}, \mathcal{B}^{ \pm}, \mathcal{C}^{ \pm}\right), a=1,2,3$, and use (7.1) and (7.2) to obtain the commutation relations

$$
\begin{aligned}
{\left[\mathcal{H}_{( \pm)}, \mathcal{F}_{a}^{ \pm}\right] } & = \pm R_{a} \mathcal{F}_{a}^{ \pm}, \quad\left[\mathcal{F}_{a}^{-}, \mathcal{F}_{a}^{+}\right]=\mathcal{P}_{a}\left(\mathcal{H}_{( \pm)}\right), \quad R_{1}=R_{2}=4, \\
\mathcal{P}_{1} & =\left.(\eta+2 \nu+3)(\eta-2 \nu+1) P_{n_{-}}(\eta) P_{n_{-}}(\eta+4)\right|_{\eta=\mathcal{H}_{(-)}-4} ^{\eta=\mathcal{H}_{(-)}}, \\
\mathcal{P}_{2} & =\left.(\eta+2 \nu+3)(\eta+2 \nu+1) P_{n_{+}}(\eta) P_{n_{+}}(\eta+4)\right|_{\eta=\mathcal{H}_{(+)}-4} ^{\eta=\mathcal{H}_{(+)}}, \\
R_{3} & =4\left(n_{n_{+}}+1\right), \quad \mathcal{P}_{3}=\left.P_{n_{+}}(\eta) P_{n_{-}}(\eta)\right|_{\eta=\mathcal{H}_{(+)}-4} ^{\eta=\mathcal{H}_{(-)}},
\end{aligned}
$$


where

$$
P_{n_{-}}(y)=\prod_{i=1}^{n_{-}}\left(y-\lambda_{i}^{-}\right), \quad P_{n_{+}}(y)=\prod_{i=1}^{n_{+}}\left(y-\lambda_{i}^{+}\right),
$$

and $\lambda_{i}^{ \pm}$are the corresponding eigenvalues of the seed states in the positive and negative schemes. Equations (7.7) are three different but related copies of the nonlinearly deformed conformal algebra $\mathfrak{s} \mathfrak{l}(2, \mathbb{R})$. One can verify that due to the nonlinearity of these three copies, the commutator of generators with different values of index $a$ do not vanish, and therefore the complete structure is rather complicated.

Similarly to the nondeformed case, be means of a unitary transformation produced by $U=e^{-i t \mathcal{H}_{( \pm)}}$we obtain the integrals of motion $\mathcal{F}_{a}^{ \pm}(t)=e^{\mp R_{a}} \mathcal{F}_{a}$, and by linear combinations of them construct the Hermitian generators $\mathfrak{D}_{a}(t)=\left(\mathcal{F}_{a}^{-}(t)-\mathcal{F}_{a}^{+}(t)\right) /\left(i 2 R_{a}\right)$ and $\boldsymbol{\Re}_{a}(t)=\left(\mathcal{F}_{a}^{+}(t)+\right.$ $\left.\mathcal{F}_{a}^{-}(t)+2 \mathcal{H}_{( \pm)}\right) / R_{a}^{2}$ which generate three copies of a nonlinear deformation of the Newton-Hooke algebra,

$$
\begin{aligned}
{\left[\mathcal{H}_{( \pm)}, \mathfrak{D}_{a}\right] } & =-i\left(\mathcal{H}_{( \pm)}-\frac{\left(R_{a}\right)^{2}}{2} \mathfrak{\Re}_{a}\right), \\
{\left[\mathcal{H}_{( \pm)}, \mathfrak{\Re}_{a}\right] } & =-2 i \mathfrak{D}_{a} \\
{\left[\mathfrak{D}_{a}, \mathfrak{\Re}_{a}\right] } & =\frac{1}{i R_{a}^{3}}\left(\mathcal{P}_{a}\left(\mathcal{H}_{( \pm)}\right)-2 R_{a} \mathcal{H}_{( \pm)}+R_{a}^{3} \mathfrak{\Re}_{a}\right),
\end{aligned}
$$

which are hidden symmetries of the system described by $\mathcal{H}_{( \pm)}$.

In the isospectral case, the operators $\mathcal{A}^{ \pm}$are the spectrum generating ladder operators, where their action on physical eigenstates of $\mathcal{H}_{( \pm)}$is similar to that of $\mathcal{C}_{\nu}^{ \pm}$in the AFF model. On the other hand, in rationally extended gapped systems obtained by Darboux transformations based on the schemes not containing Jordan states, the separated states have the form $A_{(-)}^{-} \tilde{\psi}_{-\nu-1,-l_{j}}=A_{(+)}^{-} \psi_{\nu, n_{n+}-l_{j}}$, where the states $\psi_{-\nu-1,-l_{j}}$ belong to the negative scheme and $\psi_{\nu, n_{n+}-l_{j}}$ are the omitted states in the corresponding dual positive scheme. Since by construction the separated states belong to the kernel of $A_{(-)}^{+}$, the operators $\mathcal{A}^{ \pm}$and $\mathcal{C}^{-}$will always annihilate all them.

The resulting total picture related to the action of the ladder operators can be summarized as follows. Operators of the $\mathcal{A}^{ \pm}$type detect all the separated states organized in valence bands, while they act like ordinary ladder operators in the equidistant part of the spectrum. The lowering operator $\mathcal{B}^{-}$annihilates the lowest state in each valence band, and the raising operator $\mathcal{B}^{+}$annihilates there the highest states, and $\mathcal{B}^{ \pm}$also act in an ordinary way in the equidistant part. The operators $\mathcal{C}^{ \pm}$connect the separated part of the spectrum with its equidistant part, and the lowering operator $\mathcal{C}^{-}$annihilates all the separated states as well as some excited states in the equidistant part according to the rule: if there is no level in the spectrum of energy $E_{n}-\Delta E\left(n_{n_{+}}+1\right)$, where $\Delta E=4$, then the corresponding physical eigenstate of energy $E_{n}$ is annihilated by it. For the case of the confluent Darboux transformations produced on the base of the scheme (6.8) and its dual one, the separated states are $A_{(-)}^{-} \tilde{\psi}_{m-\frac{1}{2},-l_{*}}=A_{(+)}^{-} \psi_{m-\frac{1}{2}, n_{N}-m-l_{*}}$, but the picture related to the action of the ladder operators is the same.

\section{APPLICATION: EXAMPLE}

In this section we will apply the machinery of the dual schemes and the construction of nonlinear deformations of the conformal algebra to a nontrivial example of rationally extended system with gaps. Remember that if we take $\nu=\mu+m$, we do the change $\psi_{-(\mu+m)-1, \pm n}$ by $\Omega_{-(\mu+m)-1, \pm(n-m)}$ with $n>m$ when $\mu \rightarrow-1 / 2$ in each of the relations that we have in the following, see Sec. V.

Consider a system generated on the base of the Darbouxdual schemes $\left(\psi_{\nu, 2}, \psi_{\nu, 3}\right) \sim\left(\psi_{\nu,-0}, \psi_{\nu,-1}, \psi_{\nu,-2}, \psi_{-\nu-1,-2}\right.$, $\left.\psi_{\nu,-3}, \psi_{-\nu-1,-3}\right)$. Here, $n_{-}=2, n_{+}=6, n_{n_{+}}=n_{n_{-}}=3$ and $n_{-}+n_{+}=2\left(n_{n_{+}}+1\right)=8=2 \Delta E$. The positive scheme, whose Wronskian is given explicitly in Appendix C, corresponds to the Krein-Adler scheme that provides us the system

$$
\mathcal{H}_{(+)}=-\frac{d^{2}}{d x^{2}}+V_{(+)}(x)
$$

whose potential $V_{(+)}$is plotted in Fig. 5. The spectrum of the system, $\mathcal{E}_{\nu, 0}=2 \nu+3, \mathcal{E}_{\nu, 1}=2 \nu+7, \quad \mathcal{E}_{\nu, n}=2 \nu+$ $4(n+2)+3, n=2, \ldots$, is characterized by the presence of the gap of the size $3 \Delta E=12$, which appears between the first and second excited states. The negative scheme generates the shifted Hamiltonian operator $\mathcal{H}_{(-)}=$ $\mathcal{H}_{(+)}-4 \Delta E$. In terms of the intertwining operators $A_{(+)}^{ \pm}$ and $A_{(-)}^{ \pm}$of the respective positive and negative schemes, the physical eigenstates of (8.1) are given by

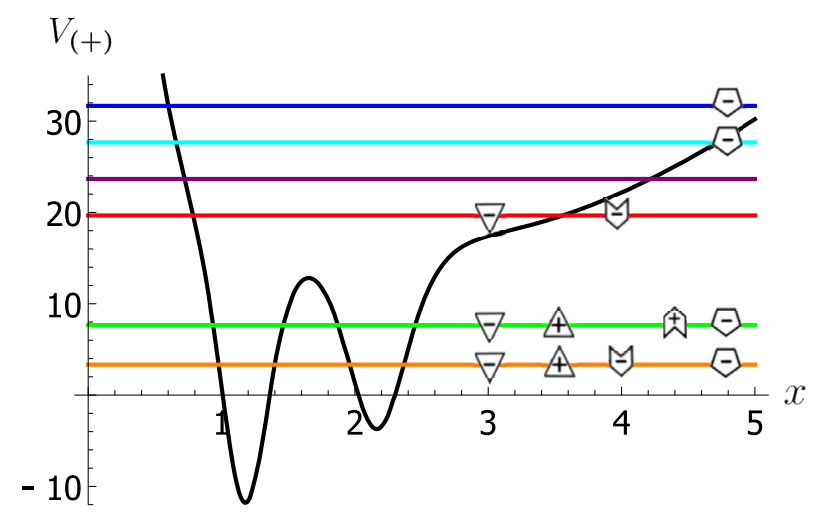

FIG. 5. The resulting potential with $\nu=1 / 3$ and energy levels of the system. The energy levels of the physical states annihilated by the ladder operators $\mathcal{A}^{-}, \mathcal{A}^{+}, \mathcal{B}^{-}, \mathcal{B}^{+}$, and $\mathcal{C}^{-}$are indicated from left to right. 


$$
\begin{gathered}
\Psi_{j}=A_{(+)}^{-} \psi_{\nu, j}=A_{(-)}^{-} \tilde{\psi}_{-\nu-1, j-3}, \\
\Psi_{j}=A_{(+)}^{-} \psi_{\nu, j+2}=A_{(-)}^{-} \psi_{\nu, j-2}, \quad j=0,1,
\end{gathered}
$$

The explicit form of the polynomials (7.8) for the system is

$$
\begin{gathered}
P_{n_{+}}(\eta)=(\eta-11-2 \nu)(\eta-15-2 \nu), \\
P_{n_{-}}(\eta)=(\eta+9-2 \nu)(\eta+13-2 \nu) \prod_{i=0}^{3}(\eta+4 n+3+2 \nu),
\end{gathered}
$$

and so, $A_{( \pm)}^{-} A_{( \pm)}^{+}=P_{n_{ \pm}}\left(\mathcal{H}_{\nu}\right)$ and $A_{( \pm)}^{-} A_{( \pm)}^{+}=P_{n_{ \pm}}\left(\mathcal{H}_{( \pm)}\right)$.

The spectrum generating ladder operators are given by Eq. (7.6), and the nonlinearly deformed conformal algebras generated by each corresponding pair of the ladder operators and the Hamiltonian $\mathcal{H}_{(+)}$are obtained from (7.7) by using polynomials (8.4) and (8.5). To clarify the physical nature of the ladder operators in more detail, we inspect their corresponding kernels by using relations (4.20) and (7.4).

We consider first the operators $\mathcal{B}^{ \pm}$, which have the lowest differential order 6 and possess the following kernels:

$$
\begin{aligned}
\operatorname{ker} \mathcal{B}^{-}= & \operatorname{span}\left\{A_{(-)}^{-} \tilde{\Psi}_{-\nu-1,-0}, A_{(+)}^{-} \tilde{\psi}_{\nu, 2}, A_{(+)}^{-} \tilde{\Psi}_{\nu, 3}, \Psi_{0},\right. \\
& \left.A_{(+)}^{-} \Psi_{-\nu-1,0}, \Psi_{2}\right\} \\
\operatorname{ker} \mathcal{B}^{+}= & \operatorname{span}\left\{A_{(+)}^{-} \tilde{\psi}_{\nu, 2}, A_{(+)}^{-} \tilde{\psi}_{\nu, 3}, A_{(+)}^{-} \psi_{\nu,-0},\right. \\
& \left.A_{(+)}^{-} \Psi_{-\nu-1,0}, \Psi_{1}, A_{(-)}^{-} \tilde{\Psi}_{-\nu-1,-1}\right\}
\end{aligned}
$$

where only $\Psi_{j}$ with $j=0,1,2$ are physical states. The operators $\mathcal{B}^{ \pm}$act like fermionic operators in the separated two-dimensional valence band, in which $\mathcal{B}^{+}$transforms the state $\Psi_{0}$ into the excited state $\Psi_{1}$, and annihilates $\Psi_{1}$, while $\mathcal{B}^{-}$acts in a similar way but in the downward direction.

The ladder operators $\mathcal{A}^{ \pm}$have differential order 14 and their kernels are

$$
\begin{aligned}
\operatorname{ker} \mathcal{A}^{-}= & \operatorname{span}\left\{A_{(-)}^{-} \tilde{\psi}_{\nu,-0}, A_{(-)}^{-} \tilde{\psi}_{\nu,-1}, A_{(-)}^{-} \tilde{\psi}_{\nu,-2}, A_{(-)}^{-} \tilde{\psi}_{\nu,-3}, \Psi_{0}, \Psi_{1}, \Psi_{2}, A_{(-)}^{-} \psi_{-\nu-1,0},\right. \\
& \left.A_{(-)}^{-} \tilde{\psi}_{-\nu-1,0}, A_{(-)}^{-} \psi_{-\nu-1,-1}, A_{(+)}^{-} \tilde{\psi}_{\nu, 1}, A_{(+)}^{-} \tilde{\psi}_{-\nu-1,1}, A_{(+)}^{-} \tilde{\psi}_{-\nu-1,2}, A_{(+)}^{-} \tilde{\psi}_{-\nu-1,3}\right\}, \\
\operatorname{ker} \mathcal{A}^{+}= & \operatorname{span}\left\{A_{(-)}^{-} \tilde{\psi}_{\nu,-0}, A_{(-)}^{-} \tilde{\psi}_{\nu,-1}, A_{(-)}^{-} \tilde{\psi}_{\nu,-2}, A_{(-)}^{-} \tilde{\psi}_{\nu,-3}, \Psi_{0}, \Psi_{1}, A_{(-)}^{-} \tilde{\psi}_{\nu, 4},\right. \\
& \left.A_{(-)}^{-} \psi_{-\nu-1,-0}, A_{(-)}^{-} \psi_{\nu,-4}, A_{(-)}^{-} \psi_{-\nu-1,-4}, A_{(-)}^{-} \tilde{\psi}_{\nu, 0}, A_{(-)}^{-} \tilde{\Psi}_{-\nu-1,0}, A_{(-)}^{-} \tilde{\psi}_{-\nu-1,1}, A_{(-)}^{-} \tilde{\psi}_{-\nu-1,2}\right\} .
\end{aligned}
$$

Both separated states are detected by $\mathcal{A}^{ \pm}$: they are annihilated by both lowering and raising ladder operators. From the described action of the ladder operators $\mathcal{A}^{ \pm}$and $\mathcal{B}^{ \pm}$it is clear that one cannot connect with their help the two separated states with the states in the equidistant part of the spectrum, and we need another pair of the ladder operators to do this job. Fortunately, we have the operators $\mathcal{C}^{ \pm}$of differential order 8, whose kernels are

$$
\begin{aligned}
\operatorname{ker} \mathcal{C}^{-}= & \operatorname{span}\left\{A_{(-)}^{-} \tilde{\psi}_{\nu,-0}, A_{(-)}^{-} \tilde{\psi}_{\nu,-1}, A_{(-)}^{-} \tilde{\psi}_{\nu,-2}, A_{(-)}^{-} \tilde{\psi}_{\nu,-3},\right. \\
& \left.\Psi_{0}, \Psi_{1}, \Psi_{4}, \Psi_{5}\right\}, \\
\operatorname{ker} \mathcal{C}^{+}= & \operatorname{span}\left\{A_{(+)}^{-} \tilde{\psi}_{\nu, 2}, A_{(+)}^{-} \tilde{\psi}_{\nu, 3}, A_{(+)}^{-} \psi_{\nu,-0}, A_{(+)}^{-} \psi_{\nu,-1},\right. \\
& \left.A_{(+)}^{-} \psi_{\nu,-2} A_{(+)}^{-} \psi_{-\nu-1,-2}, A_{(+)}^{-} \psi_{\nu,-3}, A_{(+)}^{-} \psi_{-\nu-1,-3},\right\} .
\end{aligned}
$$

These equations show that the lowering operator $\mathcal{C}^{-}$ annihilates all the states in the separated valence band as well as some states in the equidistant part of the spectrum, but the raising operator $\mathcal{C}^{+}$does not annihilate any physical state. In fact, by using the commutation relation for these operators given in (7.6), which for this case is read as $\left[\mathcal{H}_{(+)}, \mathcal{C}^{ \pm}\right]= \pm 4 \Delta E \mathcal{C}^{ \pm}$, one can derive that $\mathcal{C}^{+} \Psi_{0}=\Psi_{2}$ and $\mathcal{C}^{+} \Psi_{1}=\Psi_{3}$. This allows us to connect the separated states with the equidistant part of the spectrum.

\section{SUMMARY, DISCUSSION, AND OUTLOOK}

We studied the effect of the Klein four-group $K_{4}$ as a symmetry of the time-dependent Schrödinger equation of the AFF model, and found that it has deep implications in dependence on the values of the parameter $\nu$ in the coupling constant $g(\nu)=\nu(\nu+1) \geq-1 / 4$. In general, the action of the $K_{4}$ transformations changes the values of the energy, and transforms physical eigenstates into nonphysical ones. In the case $\nu=\ell-1 / 2$ with $\ell=0,1,2, \ldots$, however, the reflection symmetry of the coupling constant, $\rho_{1}: \nu \rightarrow-\nu-1$, reduces to the identity transformation when $\ell=0$, while for $\ell \geq 1$ it annihilates the first $\ell$ states and transforms all other eigenstates, $\rho_{1}\left(\psi_{\ell-1 / 2, n+l}\right)=(-1)^{\ell} \psi_{\ell-1 / 2, n}$, coherently with lowering their energies so that the spectrum is not changed. Thus, the $\rho_{1}$ appears to be a true, nontrivial $\mathbb{Z}_{2}$ symmetry of the system when the parameter $\nu$ takes halfinteger values. Omitting the normalization constants in wave functions $\psi_{\ell-1 / 2, n}, n=0, \ldots, \ell-1, \ell \geq 1$, annihilated by $\rho_{1}$, the same transformation allows us to construct nonphysical states which play an important role in our constructions 
and can be transformed into physical states by the action of the raising operator. On the other hand, the spatial Wick rotation $\rho_{2}:\left(x, E_{\nu, n}\right) \rightarrow\left(i x,-E_{\nu, n}\right)$ corresponds to the automorphism of the conformal algebra $\mathfrak{g l}(2, \mathbb{R})$. This automorphism transforms an infinite-dimensional unitary representation of $\mathfrak{g} \mathfrak{l}(2, \mathbb{R})$ restricted from below, which is realized on the spectrum of the AFF system, into an infinitedimensional unitary representation restricted from above. ${ }^{6}$ The nonphysical states generated by the transformations of the $K_{4}$ group appear in the kernels of the degrees of the decreasing and increasing generators of $\mathfrak{g l}(2, \mathbb{R})$. In special cases of $\nu=\ell-1 / 2$, some of those nonphysical states are changed for Jordan states of the AFF system.

We showed that in the $\mathcal{N}=2$ super-extensions of the AFF model, the nonphysical eigenstates produced by the $K_{4}$ generators play a key role in the corresponding Darboux transformations, and that the $K_{4}$ is the automorphism group of their superconformal $\mathfrak{o} \mathfrak{g} \mathfrak{p}(2,2)$ dynamical symmetry. The interesting feature of the transformations generated by the $\rho_{1}$ is that they change the systems with exact (unbroken) $\mathcal{N}=2$ Poincaré supersymmetry into the systems in a spontaneously broken phase, and vice versa. The peculiarity of the case $\nu=-1 / 2$ reveals itself here once again: both possible supersymmetric extensions of the AFF model turn out to be related by a unitary transformation provided by $\rho_{1}$, and are described by the unbroken $\mathcal{N}=2$ Poincaré supersymmetry.

Then we use the discrete transformations generated by the Klein four-group together with the conformal symmetry to generate, by means of Darboux transformations, infinite families of new, exactly solvable quantum systems with arbitrary number of gaps introduced into the equidistant spectrum of the AFF model. The obtained systems are described by symmetries of the type of finite $W$ algebras, which represent nonlinearly deformed and extended forms of the conformal $\mathfrak{g l}(2, \mathbb{R})$ algebra.

To construct such rational deformations of the AFF system and study their spectral properties, we developed the algorithm of the dual Darboux schemes for the conformal mechanics model with arbitrary values of the statistics parameter $\nu$. Note that the simplest form of the dual Darboux schemes appears in the construction of the $\mathcal{N}=2$ super-extensions of the AFF model and generators of their superconformal $\mathfrak{o} \mathfrak{g} \mathfrak{p}(2,2)$ symmetry. The physical eigenstates together with nonphysical ones generated from them by transformations of the Klein fourgroup form a base for the Darboux duality in the case of $\nu \neq \mathbb{Z}+1 / 2$. In the case of half-integer values of $\nu$ the Jordan states naturally enter the construction via the confluent Darboux transformations. Each pair of the dual

\footnotetext{
${ }^{6}$ The restricted from above unitary representations are nonphysical from the point of view of the AFF system, but both types of the $\mathfrak{s l}(2, \mathbb{R})$-representations find applications in the theory of anyons [65-67].
}

Darboux transformations different from that we employed in the construction of the $\mathcal{N}=2$ super-extensions of the AFF model, generates its some rational deformation. The obtained in such a way system can be completely isospectral (up to a global spectral shift) to the initial conformal mechanics model, or may have a finite number of valence bands in the low part of the spectrum, which are separated by gaps between themselves and from the semiinfinite band with equidistant energy levels. The minimal size of a gap in our construction corresponds to one missing energy level in comparison with two missing levels in gaps of minimal size in the systems generated by the KreinAdler transfromations, which also are included in our dual Darboux schemes.

We showed that when the statistics parameter varies continuously, the spectrum of rationally deformed AFF systems suffers structural changes at half-integer ("fermionic") values of $\nu$. No such changes happen, however, at integer values of $\nu$ corresponding to the case of bosons in the context of the statistics transmutations [29-31]. Recall that all the deformations of the conformal mechanics model with $\nu \in \mathbb{Z}$ can be generated by generalized Darboux transformations from the quantum harmonic oscillator system [42]. At the same time we also note here that the question of the self-adjoint extension of the AFF Hamiltonian operator requires a special consideration in the case $\nu=-1 / 2$ [54], which corresponds to a minimal value of the coupling constant $g$ for which the spectrum is bounded from below, and when, as we saw, the Klein four-group symmetry suffers minimal breaking.

The Darboux duality allowed us to obtain the set of the three pairs of ladder operators of different but complementary nature. In the case of the rationally deformed gapped systems, Hermitian conjugate ladder operators of the $\mathcal{A}$ type detect all the separated states, each of which is annihilated by both, the lowering and the raising, operators; the lowering operator $\mathcal{A}^{-}$also annihilates the lowest state in the equidistant part of the spectrum. The raising ladder operator of the $\mathcal{B}$ type detects the states with highest energy level in each valence band by annihilating them. The lowering operator $\mathcal{B}^{-}$makes the same with the states of the lowest energy level in each valence band, and also annihilates the lowest state in the equidistant part of the spectrum. Although the operators of these two types detect all the separated states as well as identify the borders of the valence bands and the edge of the semi-infinite band with equidistant energy levels, they cannot connect the states from different bands. This job is realized with the help of the ladder operators of the $\mathcal{C}$ type. As a result, one can see that any of the two sets of the ladder operators, $\left(\mathcal{C}^{ \pm}, \mathcal{A}^{ \pm}\right)$or $\left(\mathcal{C}^{ \pm}, \mathcal{B}^{ \pm}\right)$, forms a complete set of the spectrum generating ladder operators by which any eigenstate of the rationally deformed AFF system can be transformed into its any other eigenstate. In the case if we have an isospectral deformation of the AFF system $\mathcal{H}_{\nu}$ obtained via the Darboux scheme 
(6.3), the operators $\mathcal{A}^{ \pm}$are enough to generate the entire tower of physical eigenstates starting from any physical eigenstate.

Each of the three pairs of the conjugate ladder operators together with the Hamiltonian operator generate some nonlinearly deformed version of the conformal $\mathfrak{g l}(2, \mathbb{R})$ algebra of the $W$-type [68], which is the symmetry of the corresponding rationally deformed AFF system of a generic form. We, however, did not compute commutators between ladder operators of different types, but with a quick inspection one can notice that new structures are generated. Though the resulting picture is expected to be rather complicated and requires a separate study, it should be similar to that appearing in the case of $\nu=0$, which was analyzed in detail in [43], as well as to that in the $P T$-regularized two-particle Calogero systems $[69,70]$, and can be described as follows. Any extended system composed from a pair of the AFF systems characterized by the parameters $\nu$ and $\nu+m, m \in \mathbb{Z}$, are described, as we showed, by the superconformal $\mathfrak{o} \mathfrak{g} \mathfrak{p}(2,2)$ symmetry in the case of $m=1$, while a nonlinear deformation of this superalgebra should appear when $m>0$. On the other hand, if the composed system contains a pair of rationally deformed AFF systems, according to our results in [43], one can expect that its spectrum should be described by some nonlinear extensions of the $\mathfrak{o} \mathfrak{g p}(2 \mid 2)$ symmetry. Some nonlinearly extended versions of $\mathfrak{g l}(2, \mathbb{R})$ are expected then to appear as $W$-type algebras describing symmetries of the rationally deformed AFF systems.

Our consideration of rational deformations of the conformal mechanics was restricted by inclusion of Jordan states of the simplest form. Following the analysis and ideas presented in Refs. [44-47,49], the constructions can be generalized to the case of higher order Jordan states defined via relations

$$
\begin{aligned}
\left(L-\lambda_{*}\right) \Omega_{*}^{(0)} & =\psi_{*}, \quad\left(L-\lambda_{*}\right) \Omega_{*}^{(k)}=\Omega_{*}^{(k-1)}, \\
k & =1, \ldots,
\end{aligned}
$$

as well as to their further generalizations defined as the states annihilated by polynomial in $L$ operators [48]. The states that we have used correspond to $\Omega_{*}^{(0)}$. In this way, one can produce the systems by means of confluent Darboux transformations which involve more Jordan states of these chains, and one can expect that the spectrum of the resulting systems will have a similar gapped structure. Then it would be interesting to study such kind of the systems from the point of view of the spectrum generating ladder operators and the extended nonlinear deformations of the (super)conformal algebra associated with them.

It is known that the conformal symmetry underlies the relation between the quantum free particle and harmonic oscillator systems $[17,71,72]$. A similar picture also is valid for the two-particle Calogero system without confining potential term and omitted center of mass degree of freedom, i.e., for the system (3.1), and the AFF model (3.7) $[2,25,73,74]$. The Calogero model and its deformations, in turn, are intimately related to the soliton solutions of the Korteweg-de Vries equation and higher equations of its hierarchy [41,75-77]. It would be interesting to investigate the question of a possible relation between rational deformations of the AFF model studied here and solutions to the same hierarchy of completely integrable systems described by partial differential equations. At the same time, the approach based on the Klein four-group transformations employed here can also be applied to the two-particle Calogero model with arbitrary values of the statistics parameter $\nu$ but without the confining harmonic potential term. In this way one could expect to generate new quantum solvable systems which may be related to the Korteweg-de Vries hierarchy.

\section{ACKNOWLEDGMENTS}

The work was partially supported by the CONICYT scholarship 21170053 (L. I.), FONDECYT Project 1190842 (M. S. P.), and the Project USA 1899 (M. S.P. and L. I.).

\section{APPENDIX A: DUAL SCHEMES IN HALF-INTEGER CASE}

To obtain the dual schemes in the half-integer case, we analyze first the relations that exist between of $\mathcal{H}_{-1 / 2}$ and $\mathcal{H}_{-1 / 2+\ell}$. The latter are given by the dual schemes $\left(\psi_{-1 / 2, \pm 0}, \ldots, \psi_{-1 / 2, \pm(\ell-1)}\right)$, whose Wronskians are

$$
W\left(\psi_{-1 / 2, \pm 0}, \ldots, \psi_{-1 / 2, \pm(\ell-1)}\right)=x^{\ell^{2} / 2} e^{\mp \ell x^{2} / 2} .
$$

The corresponding intertwiners map eigen- and Jordan states of $\mathcal{H}_{-1 / 2}$ to those of $\mathcal{H}_{-1 / 2+\ell}$. If we choose the scheme with positive indexes, some of these mappings useful for the following are given by

$$
\begin{aligned}
\mathbb{A}_{\ell}^{-} \psi_{-1 / 2, n} & =\psi_{-1 / 2+\ell, n-\ell}, \quad \mathbb{A}_{\ell}^{-} \Omega_{\nu,-1 / 2}=\Omega_{-1 / 2+\ell, n-\ell}, \\
n & \geq \ell, \\
\mathbb{A}_{\ell}^{-} \Omega_{-1 / 2, l}=\psi_{-(-1 / 2+\ell)-1, l}, & l<\ell, \quad \text { (A3 })
\end{aligned}
$$

where $\mathbb{A}_{\bar{\ell}}^{-}$and its Hermitian conjugate $\mathbb{A}_{\ell}^{+}$are the intertwining operators of the chosen Darboux transformation. On the other hand if we take the scheme with negative sign in indices, we obtain another intertwining operators $\mathbb{B}_{\ell}^{ \pm}$, which satisfy the relation $\mathbb{B}_{\ell}^{ \pm}=(i)^{\ell} \rho_{2}\left(\mathbb{A}_{\ell}^{ \pm}\right)$, i.e., their action on eigenstates and Jordan states can be obtained by application of $\rho_{2}$ to the relations that correspond to the action of $\mathbb{A}_{m}^{ \pm}$.

Now, to derive the dual schemes let us assume that we have a collection of nonrepeated seed states of the form 
$\left(\psi_{-1 / 2,0}, \ldots, \psi_{-1 / 2, \ell-1},\left\{\vartheta_{-1 / 2}\right\}\right)$, where $\left\{\vartheta_{-1 / 2}\right\}$ contains $N_{1}$ arbitrary physical states $\psi_{-1 / 2, k_{i}}$ with $k_{i}>\ell-1$ for $i=1, \ldots, N_{1}$, and $N_{2}$ arbitrary Jordan states of the form $\Omega_{-1 / 2, l_{j}}$ with $j=1, \ldots, N_{1}$. In the same way as we did in Sec. V, we define $n_{N}$ as the largest of the numbers $n_{N_{1}}$ and $n_{N_{2}}$, and also we suppose for simplicity that the signs of both $k_{i}$ and $k_{j}$ are positive. Then we use (2.8) and (A1) to write $W\left(\psi_{-1 / 2,0}, \ldots, \psi_{1 / 2, \ell-1},\left\{\vartheta_{-1 / 2}\right\}\right)=x^{\ell^{2} / 2} e^{-\ell x^{2} / 2} W$ $\left(\left\{\mathbb{A}_{\ell}^{-} \vartheta_{-1 / 2}\right\}\right)$. The next step is to use the extension of the dual schemes for $\nu=-1 / 2$, i.e., we change each function of the form $\psi_{-\nu-1, n}$ by $\Omega_{-1 / 2, n}$ in Eq. (5.2), and use it to rewrite this last Wronskian relation as

$$
W\left(\mathbb{A}_{\ell}^{-}\left\{\vartheta_{-1 / 2}\right\}\right)=x^{-\ell^{2} / 2} e^{-\left(n_{N}+1-\ell / 2\right) x^{2}} W\left(\left\{\Delta_{-}^{(-1 / 2)}\right\}\right),
$$

where $\Delta_{-}^{(-1 / 2)}$ is the dual scheme of $\left(\psi_{-1 / 2,0}, \ldots, \psi_{-1 / 2, \ell-1}\right.$, $\left.\left\{\vartheta_{-1 / 2}\right\}\right)$ given by $\left\{\Delta_{-}^{(-1 / 2)}\right\}=\left(\psi_{-1 / 2,-0}, \ldots, \psi_{-1 / 2,-(\ell-1)}\right.$, $\left.\left\{\vartheta_{-1 / 2}^{-}\right\}\right)$, and

$$
\begin{aligned}
\left\{\vartheta_{-1 / 2}^{-}\right\}= & \left(\psi_{-1 / 2,-\ell}, \Omega_{-1 / 2,-0}, \ldots, \check{\psi}_{-1 / 2,-s_{j}}, \check{\Omega}_{-1 / 2,-r_{i}}, \ldots,\right. \\
& \left.\psi_{-1 / 2,-n_{N}}, \Omega_{-1 / 2,-n_{N}}\right) .
\end{aligned}
$$

Here, as well as in the nonhalf-integer case, the marked functions $\check{\psi}_{-1 / 2,-s_{j}}$ and $\check{\Omega}_{-1 / 2,-r_{i}}$ indicate the omitted states with $s_{j}=n_{N}-l_{j}$ and $r_{i}=n_{N}-k_{i}$. In the last step, we use Eqs. (2.8) and (A1) with the negative sign to write the equality $W\left(\left\{\Delta_{-}^{(-1 / 2)}\right\}\right)=x^{\ell^{2} / 2} e^{\ell x^{2} / 2} W\left(\mathbb{B}_{\ell}^{-}\left\{\vartheta_{-1 / 2}^{-}\right\}\right)$and as analog of (A4) we obtain

$$
\begin{aligned}
W\left(\mathbb{A}_{\ell}^{-}\left\{\vartheta_{-1 / 2}\right\}\right) & =e^{-\left(n_{N}^{\prime}+1\right) x^{2}} W\left(\mathbb{B}_{\ell}^{-}\left\{\vartheta_{-1 / 2}^{-}\right\}\right), \\
n_{N}^{\prime} & =n_{N}-\ell .
\end{aligned}
$$

This relation is the dual scheme equation for the case $\nu=\ell-1 / 2$. By means of (A2) and its analogs for $\mathbb{B}_{\ell}^{-}$ obtained by the application of $\rho_{2}$, we conclude that in the scheme of the left-hand side of the equation there are $N_{1}$ physical states of the form $\mathbb{A}_{\ell}^{-} \psi_{-1 / 2, k_{i}}=\psi_{\ell-1 / 2, k_{i}-\ell}$, and a mixture of $N_{2}$ Jordan states and formal states produced by $\rho_{2}$ distributed in the following way: we have Jordan states $\mathbb{A}_{\ell}^{-} \Omega_{-1 / 2, l_{i}}=\psi_{\ell-1 / 2, l_{i}}$ when $l_{i}<\ell-1$, and formal states $\mathbb{A}_{\ell}^{-} \Omega_{-1 / 2, l_{i}}=\psi_{\ell-1 / 2, l_{i}-\ell}$ when $l_{i} \geq \ell$. The omitted states in the scheme on the right-hand side are $\mathbb{B}_{\ell}^{-} \check{\psi}_{-1 / 2,-s_{j}}=\check{\psi}_{-1 / 2+\ell,-\left(s_{j}-\ell\right)}$ and $\mathbb{B}_{\ell}^{-} \check{\Omega}_{-1 / 2,-r_{j}}=\check{\psi}_{-\ell-1 / 2,-r_{j}}$ $\left(\mathbb{B}_{\ell}^{-} \check{\Omega}_{-1 / 2,-r_{j}}=\check{\psi}_{-\ell-1 / 2,-\left(r_{j}-\ell\right)}\right)$ when $r_{j} \leq \ell-1\left(r_{j}>\ell\right)$. Note that the largest index in both sides of the equation is now given by $n_{N}^{\prime}=n_{N}-\ell$. In comparison with the nonhalf-integer case, this is the same result that we would obtain if we consider Eq. (5.2) in the nonhalf-integer case, and then formally change the states of the form $\psi_{-\nu-1, l_{i}}$ by $\Omega_{-\ell-1 / 2, l_{i}-\ell}$ when $l_{i} \geq \ell$ in the limit $\nu \rightarrow \ell-1 / 2$.
Relation analogous to (5.3) would be obtained if we start from the case $\nu=-1 / 2$ with a scheme composed from the eigenstates and Jordan states produced by $\rho_{2}$, and then apply the same arguments employed for the case analyzed above.

\section{APPENDIX B: SOME WRONSKIAN RELATIONS}

We show here that the Wronskian (6.5) takes nonzero values and that it reduces to (6.8) in the limit $\mu \rightarrow-1 / 2$. For this, consider first a generic system (2.1) which has a set of the seed states $\left(\phi_{1}, \phi_{2}, \ldots, \phi_{2 l-1}, \phi_{2 l}\right)$ with eigenvalues $\lambda_{1}<\lambda_{2}<\ldots<\lambda_{2 l-1}<\lambda_{2 l}$. Then the following relation

$W\left(\phi_{1}, \phi_{2}, \ldots, \phi_{2 l-1}, \phi_{2 l}\right)=\prod_{i=0}^{l-1} W\left(\mathbb{A}_{2 i} \phi_{2 i+1}, \mathbb{A}_{2 i} \phi_{2 i+2}\right)$,

can be proved by induction, where $\mathbb{A}_{0}=1$, and $\mathbb{A}_{2 i}$ with $i \geq 1$ corresponds to the intertwining operator associated with the scheme $\left(\phi_{1}, \ldots, \phi_{2 i}\right)$. From (B1) it follows that if each factor $W\left(\mathbb{A}_{2 i} \phi_{2 i+1}, \mathbb{A}_{2 i} \phi_{2 i+2}\right)$ does not have zeros, then the complete Wronskian neither has them. To inspect the properties of the Wronskian factors, we use the relation

$W^{\prime}\left(\mathbb{A}_{2 i} \phi_{2 i+1}, \mathbb{A}_{2 i} \phi_{2 i+2}\right)=\left(\lambda_{2 i+2}-\lambda_{2 i+1}\right) \mathbb{A}_{2 i} \phi_{2 i+1} \mathbb{A}_{2 i} \phi_{2 i+2}$,

and integrate it from $a$ to $x$,

$$
\begin{aligned}
& W\left(\mathbb{A}_{2 i} \phi_{2 i+1}, \mathbb{A}_{2 i} \phi_{2 i+2}\right) \\
& =\left(\lambda_{2 i+2}-\lambda_{2 i+1}\right) \int_{a}^{x} \mathbb{A}_{2 i} \phi_{2 i+1} \mathbb{A}_{2 i} \phi_{2 i+2} d \zeta+\omega,
\end{aligned}
$$

where $\omega=\left.W\left(\mathbb{A}_{2 i} \phi_{2 i+1}, \mathbb{A}_{2 i} \phi_{2 i+2}\right)\right|_{x=a}$. In the case when functions $\mathbb{A}_{2 i} \phi_{2 i+1}, \mathbb{A}_{2 i} \phi_{2 i+2}$ and their first derivatives vanish in $b$, we find $\omega=-\left(\lambda_{2 i+2}-\lambda_{2 i+1}\right) \int_{a}^{b} \mathbb{A}_{2 i} \phi_{2 i+1}$ $\mathbb{A}_{2 i} \phi_{2 i+2} d \zeta$, and then

$$
\begin{aligned}
& W\left(\mathbb{A}_{2 i} \phi_{2 i+1}, \mathbb{A}_{2 i} \phi_{2 i+2}\right) \\
& =-\left(\lambda_{2 i+2}-\lambda_{2 i+1}\right) \int_{x}^{b} \mathbb{A}_{2 i} \phi_{2 i+1} \mathbb{A}_{2 i} \phi_{2 i+2} d \zeta .
\end{aligned}
$$

Relation (B1) takes then the form

$$
\begin{aligned}
& W\left(\phi_{1}, \phi_{2}, \ldots, \phi_{2 l-1}, \phi_{2 l}\right) \\
& \quad=\prod_{i=0}^{l-1}\left(\lambda_{2 i+1}-\lambda_{2 i+2}\right) \int_{x}^{b} \mathbb{A}_{2 i} \phi_{2 i+1} \mathbb{A}_{2 i} \phi_{2 i+2} d \zeta_{i} .
\end{aligned}
$$

Analogously, one can consider a generic system, choose $l$ solutions $\varphi_{i}$ of Eq. (2.1), and construct $l$ corresponding Jordan states $\Omega_{i}$ using Eq. (2.13). Assuming also that these states satisfy relations (2.16), one can find that 


$$
\begin{aligned}
W\left(\varphi_{1}, \Omega_{1}, \ldots, \varphi_{l}, \Omega_{l}\right) & =\prod_{i=0}^{l-1} W\left(\mathbb{A}_{2 i}^{\Omega} \varphi_{i+1}, \mathbb{A}_{2 i}^{\Omega} \Omega_{i+1}\right) \\
& =\prod_{i=0}^{l-1} \int_{x}^{b}\left(\mathbb{A}_{2 i}^{\Omega} \varphi_{i+1}\right)^{2} d \zeta_{i}
\end{aligned}
$$

where $\mathbb{A}_{0}^{\Omega}=1$ and $\mathbb{A}_{2 i}^{\Omega}$ correspond to the intertwining operator associated with the scheme $\left(\varphi_{1}, \Omega_{1} \ldots, \varphi_{l}, \Omega_{l}\right)$. Relation (B6) can be proved in a way similar to that for (B5).

Let us turn now to the AFF model, where $a=0, b=\infty$, and choose the seed states in (B1) in correspondence with our picture: for $i=0, \ldots, l-1$ we fix $\phi_{2 i+1}=\psi_{-\mu-m-1, n_{i+1}}$ and $\phi_{2 i+2}=\psi_{\mu+m, n_{i+1}-m}$. This identification implies that $\lambda_{2 i+1}=E_{-\mu-m-1, n_{i+1}}, \quad \lambda_{2 i+2}=E_{\mu+m, n_{i+1}-m}, \quad$ and $\lambda_{2 i+2}-$ $\lambda_{2 i+1}=4(\mu+1 / 2)$. These both functions and their first derivatives behave for large values of $x$ as $e^{-x^{2} / 2}$, and vanish at $x=\infty$. This behavior is not changed by application of any differential operator with which we work. On the other hand, near zero we have $\mathbb{A}_{2 i} \psi_{-\mu+m+1, n_{i+1}} \sim$ $x^{-\mu-m-i}$ and $\mathbb{A}_{2 i} \psi_{\mu+m, n_{i+1}-m} \sim x^{\mu+m+1+i}$. Therefore, for small values of $x, \mathbb{A}_{2 i} \psi_{-\mu+m+1, n_{i+1}} \mathbb{A}_{2 i} \psi_{\mu+m, n_{i+1}-m} \sim x$, and $W\left(\mathbb{A}_{2 i} \psi_{-\mu+m+1, n_{i+1}}, \mathbb{A}_{2 i} \psi_{\mu+m, n_{i+1}-m}\right)$ takes a finite value when $x \rightarrow 0^{+}$. Knowing this and Eq. (B2), we employ the Adler method [38], and use the theorem on nodes of wave functions to show that zeros and the minima and maxima of the functions $\mathbb{A}_{2 i} \psi_{-\mu+m+1, n_{i+1}}$ and $\mathbb{A}_{2 i} \psi_{\mu+m, n_{i+1}-m}$ do not coincide, and that their corresponding Wronskian is nonvanishing.

In the case $\mu=-1 / 2$, we put $\varphi_{j}=\psi_{m-1 / 2, n_{j+1}-n}$ with $j=0, \ldots, l-1$, and then we arrive at the relations

$$
\begin{aligned}
\frac{W\left(\left\{\gamma_{\mu}\right\}\right)}{(4 \mu+2)^{N}}= & (-1)^{l} \prod_{i=0}^{l-1} \int_{x}^{\infty} \mathbb{A}_{2 i} \psi_{-\mu-m-1, n_{i+1}} \\
& \times \mathbb{A}_{2 i} \psi_{\mu+m, n_{n_{i+1}}-m} d \zeta_{i},
\end{aligned}
$$

$$
W(\{\gamma\})=\prod_{j=0}^{l-1} \int_{x}^{\infty}\left(\mathbb{A}_{2 j}^{\Omega} \psi_{m-1 / 2, n_{j+1}-m}\right)^{2} d \zeta_{j}
$$

where the sets $\left\{\gamma_{\mu}\right\}$ and $\{\gamma\}$ are defined in (6.5) and (6.8). We note that both equations are pretty similar each other, and if we suppose that $\mathbb{A}_{2 i} \rightarrow \mathbb{A}_{2 i}^{\Omega}$ when $\mu \rightarrow-1 / 2$, and take into account the relation $\psi_{m-1 / 2, n_{j}-m} \propto \psi_{-(m-1 / 2)-1, n_{j}}$, we find that

$$
\lim _{\mu \rightarrow-1 / 2} \frac{W\left(\left\{\gamma_{\mu}\right\}\right)}{(4 \mu+2)^{N}} \propto W(\{\gamma\}) .
$$

This relation is true for the case $i=1$, which implies that $\mathbb{A}_{2} \rightarrow \mathbb{A}_{2}^{\Omega}$ in the corresponding limit. The general case is proved by induction.

\section{APPENDIX C: WRONSKIANS (5.8), (5.9)}

The explicit form of the Wronskians (5.8) and (5.9), which are used in the main text, is

$$
\begin{aligned}
& W\left(\psi_{\nu, 2}, \psi_{-\nu-1,2}\right) \\
& =(2 \nu+1) e^{-x^{2}}\left(45-72 \nu+16\left(-4 x^{6}+x^{8}\right)\right. \\
& \left.\quad+8 x^{4}(15-4 \nu(1+\nu))+\nu^{2}(-7+2 \nu(2+\nu))\right),
\end{aligned}
$$

$$
\begin{aligned}
& W\left(\psi_{\nu, 2}, \psi_{\nu, 3}\right) \\
& =e^{-x^{2}} x^{3+2 \nu}\left(16 x^{8}-32 x^{6}(5+2 \nu)+24 x^{4}(5+2 \nu)^{2}\right. \\
& \quad-8 x^{2}(3+2 \nu)(5+2 \nu)(7+2 \nu)+(3+2 \nu)(5+2 \nu)^{2} \\
& \quad \times(7+2 \nu)) .
\end{aligned}
$$

[1] K. Sundermeyer, Symmetries in Fundamental Physics, Fundam. Theor. Phys. Vol. 176 (Springer, Dordrecht, 2014), https://doi.org/10.1007/978-94-007-7642-5.

[2] V. de Alfaro, S. Fubini, and G. Furlan, Conformal invariance in quantum mechanics, Nuovo Cimento Soc. Ital. Fis. 34A, 569 (1976).

[3] F. Calogero, Solution of a three-body problem in onedimension, J. Math. Phys. (N.Y.) 10, 2191 (1969); Solution of the one-dimensional $\mathrm{N}$ body problems with quadratic and/or inversely quadratic pair potentials, J. Math. Phys. (N.Y.) 12, 419 (1971).

[4] I. I. Goldman and V. D. Krivchenkov, Problems in Quantum Mechanics (Addison-Wesley, Reading, MA, 1961).
[5] V. P. Akulov and A. I. Pashnev, Quantum superconformal model in $(1,2)$ space, Theor. Math. Phys. 56, 862 (1983).

[6] S. Fubini and E. Rabinovici, Superconformal quantum mechanics, Nucl. Phys. B245, 17 (1984).

[7] E. A. Ivanov, S. O. Krivonos, and V. M. Leviant, Geometric superfield approach to superconformal mechanics, J. Phys. A 22, 4201 (1989).

[8] E. E. Donets, A. Pashnev, V. O. Rivelles, D. P. Sorokin, and M. Tsulaia, $N=4$ superconformal mechanics and the potential structure of AdS spaces, Phys. Lett. B 484, 337 (2000).

[9] F. Fedoruk, E. Ivanov, and O. Lechtenfeld, Superconformal mechanics, J. Phys. A 45, 173001 (2012). 
[10] A. Galajinsky, Geometry of the isotropic oscillator driven by the conformal mode, Eur. Phys. J. C 78, 72 (2018).

[11] P. Claus, M. Derix, R. Kallosh, J. Kumar, P. K. Townsend, and A. Van Proeyen, Black Holes and Superconformal Mechanics, Phys. Rev. Lett. 81, 4553 (1998).

[12] J. Michelson and A. Strominger, Superconformal multiblack hole quantum mechanics, J. High Energy Phys. 09 (1999) 005.

[13] J. A. de Azcárraga, J. M. Izquierdo, J. C. P. Bueno, and P. K. Townsend, Superconformal mechanics, black holes, and nonlinear realizations, Phys. Rev. D 59, 084015 (1999).

[14] G. W. Gibbons and P. K. Townsend, Black holes and Calogero models, Phys. Lett. B 454, 187 (1999).

[15] R. Britto-Pacumio, J. Michelson, A. Strominger, and A. Volovich, Lectures on superconformal quantum mechaics and multi-black hole moduli spaces, NATO Sci. Ser. C 556, 255 (2000).

[16] A. Galajinsky, $\mathcal{N}=4$ superconformal mechanics from the $s u(2)$ perspective, J. High Energy Phys. 02 (2015) 091.

[17] C. Duval, G. W. Gibbons, and P. Horvathy, Celestial mechanics, conformal structures and gravitational waves, Phys. Rev. D 43, 3907 (1991).

[18] B. Pioline and A. Waldron, Quantum Cosmology and Conformal Invariance, Phys. Rev. Lett. 90, 031302 (2003).

[19] M. Cariglia, A. Galajinsky, G. W. Gibbons, and P. A. Horvathy, Cosmological aspects of the Eisenhart-Duval lift, Eur. Phys. J. C 78, 314 (2018).

[20] D. T. Son, Toward an AdS/could atoms correspondence: A geodesic realization of Schrodinger symmetry, Phys. Rev. D 78, 046003 (2008).

[21] K. Balasubramanian and J. McGreevy, Gravity Duals for Nonrelativistic CFTs, Phys. Rev. Lett. 101, 061601 (2008).

[22] J. L.F. Barbon and C. A. Fuertes, On the spectrum of nonrelativistic AdS/CFT, J. High Energy Phys. 09 (2008) 030 .

[23] C. Chamon, R. Jackiw, S. Y. Pi, and L. Santos, Conformal quantum mechanics as the $\mathrm{CFT}_{1}$ dual to $\mathrm{AdS}_{2}$, Phys. Lett. B 701, 503 (2011).

[24] T. Okazaki, Whittaker vector, Wheeler-DeWitt equation, and the gravity dual of conformal quantum mechanics, Phys. Rev. D 92, 126010 (2015).

[25] S. Brodsky, G. de Teramond, H. Gunter, and J. Erlich, Lightfront holographic QCD and emerging confinement, Phys. Rep. 584, 1 (2015).

[26] A. Deur, S. J. Brodsky, and G. F. de Teramond, Connecting the hadron mass scale to the fundamental mass scale of quantum chromodynamics, Phys. Lett. B 750, 528 (2015).

[27] A. Prain, S. Fagnocchi, and S. Liberati, Analogue cosmological particle creation: Quantum correlations in expanding Bose-Einstein condensates, Phys. Rev. D 82, 105018 (2010).

[28] K. Ohashi, T. Fujimori, and M. Nitta, Conformal symmetry of trapped Bose-Einstein condensates and massive NambuGoldstone modes, Phys. Rev. A 96, 051601 (2017).

[29] J. M. Leinaas and J. Myrheim, On the theory of identical particles, Nuovo Cimento Soc. Ital. Fis. 37B, 1 (1977); Intermediate statistics for vortices in superfluid films, Phys. Rev. B 37, 9286 (1988).
[30] R. MacKenzie and F. Wilczek, Peculiar spin and statistics in two space dimensions, Int. J. Mod. Phys. A 03, 2827 (1988).

[31] A. P. Polychronakos, Physics and mathematics of Calogero particles, J. Phys. A 39, 12793 (2006).

[32] G. Darboux, Sur une proposition relative aux équations linéaires, C.R. Hebd. Seances Acad. Sci. 94, 1456 (1882).

[33] M. M. Crum, Associated Sturm-Liouville systems, Q. J. Math. 6, 121 (1955).

[34] V. B. Matveev and M. A. Salle, Darboux Transformations and Solitons (Springer, Berlin, 1991), https://www.springer .com/gp/book/9783662009246.

[35] E. Witten, Dynamical breaking of supersymmetry, Nucl. Phys. B188, 513 (1981); Constraints on supersymmetry breaking, Nucl. Phys. B202, 253 (1982).

[36] F. Cooper, A. Khare, and U. Sukhatme, Supersymmetry and quantum mechanics, Phys. Rep. 251, 267 (1995).

[37] M. G. Krein, On a continuous analogue of a Christoffel formula from the theory of orthogonal polynomials, Dokl. Akad. Nauk SSSR 113, 970 (1957).

[38] V. E. Adler, A modification of Crum's method, Theor. Math. Phys. 101, 1381 (1994).

[39] A. Arancibia, J. M. Guilarte, and M. S. Plyushchay, Effect of scalings and translations on the supersymmetric quantum mechanical structure of soliton systems, Phys. Rev. D 87, 045009 (2013).

[40] A. Arancibia and M. S. Plyushchay, Chiral asymmetry in propagation of soliton defects in crystalline backgrounds, Phys. Rev. D 92, 105009 (2015).

[41] J. M. Guilarte and M.S. Plyushchay, Perfectly invisible PT-symmetric zero-gap systems, conformal field theoretical kinks, and exotic nonlinear supersymmetry, J. High Energy Phys. 12 (2017) 061.

[42] J. F. Cariñena, L. Inzunza, and M. S. Plyushchay, Rational deformations of conformal mechanics, Phys. Rev. D 98, 026017 (2018).

[43] L. Inzunza and M. S. Plyushchay, Hidden symmetries of rationally deformed superconformal mechanics, Phys. Rev. D 99, 025001 (2019).

[44] A. Schulze-Halberg, Wronskian representation for confluent supersymmetric transformation chains of arbitrary order, Eur. Phys. J. Plus 128, 68 (2013).

[45] F. Correa, V. Jakubsky, and M. S. Plyushchay, PT-symmetric invisible defects and confluent Darboux-Crum transformations, Phys. Rev. A 92, 023839 (2015).

[46] A. Contreras-Astorga and A. Schulze-Halberg, On integral and differential representations of Jordan chains and the confluent supersymmetry algorithm, J. Phys. A 48, 315202 (2015).

[47] D. Bermudez, Wronskian differential formula for k-confluent SUSY QM, Ann. Phys. (Amsterdam) 364, 35 (2016).

[48] J. F. Cariñena and M. S. Plyushchay, Ground-state isolation and discrete flows in a rationally extended quantum harmonic oscillator, Phys. Rev. D 94, 105022 (2016).

[49] A. Schulze-Halberg, Regularity conditions for transformed potentials in the confluent supersymmetry algorithm, Int. J. Mod. Phys A. 33, 1850214 (2018).

[50] M. S. Plyushchay, Schwarzian derivative treatment of the quantum second-order supersymmetry anomaly, and 
coupling-constant metamorphosis, Ann. Phys. (Amsterdam) 377, 164 (2017).

[51] J.F. Cariñena and M.S. Plyushchay, ABC of ladder operators for rationally extended quantum harmonic oscillator systems, J. Phys. A 50, 275202 (2017).

[52] L. Inzunza and M. S. Plyushchay, Hidden superconformal symmetry: Where does it come from?, Phys. Rev. 97, 045002 (2018).

[53] L. D. Landau and E. M. Lifshitz, Quantum Mechanics, Course of Theoretical Physics Vol. 3 (Pergamon Press, New York, 1965).

[54] K. Kirsten and P. Loya, Spectral functions for the Schrodinger operator on $\mathbb{R}^{+}$with a singular potential, J. Math. Phys. (N.Y.) 51, 053512 (2010).

[55] A. Galajinsky, Conformal mechanics in Newton-Hooke spacetime, Nucl. Phys. B832, 586 (2010).

[56] K. Andrzejewski, Conformal Newton-Hooke algebras, Niederer's transformation and Pais-Uhlenbeck oscillator, Phys. Lett. B 738, 405 (2014).

[57] M. Asorey, J. F. Cariñena, G. Marmo, and A. Perelomov, Isoperiodic classical systems and their quantum counterparts, Ann. Phys. (Amsterdam) 322, 1444 (2007).

[58] V. Bargmann, Irreducible unitary representations of the Lorentz group, Ann. Math. 48, 568 (1947).

[59] M. S. Plyushchay, Quantization of the classical SL(2,R) system and representations of SL(2,R) group, J. Math. Phys. (N.Y.) 34, 3954 (1993).

[60] P. A. M. Dirac, Forms of relativistic dynamics, Rev. Mod. Phys. 21, 392 (1949).

[61] M. Axenides, E. G. Floratos, and S. Nicolis, Modular discretization of the $\mathrm{AdS}_{2} / \mathrm{CFT}_{1}$ holography, J. High Energy Phys. 02 (2014) 109.

[62] H. Falomir, P. A. G. Pisani, and A. Wipf, Pole structure of the Hamiltonian zeta function for a singular potential, J. Phys. A 35, 5427 (2002).

[63] H. Falomir and P. A. G. Pisani, Self-adjoint extensions and SUSY breaking in supersymmetric quantum mechanics, J. Phys. A 38, 4665 (2005).
[64] Y. Grandati, Multistep DBT and regular rational extensions of the isotonic oscillator, Ann. Phys. (Amsterdam) 327, 2411 (2012).

[65] M. S. Plyushchay, The model of relativistic particle with torsion, Nucl. Phys. B362, 54 (1991).

[66] R. Jackiw and V.P. Nair, Relativistic wave equations for anyons, Phys. Rev. D 43, 1933 (1991).

[67] P. A. Horvathy, M. S. Plyushchay, and M. Valenzuela, Bosons, fermions and anyons in the plane, and supersymmetry, Ann. Phys. (Amsterdam) 325, 1931 (2010).

[68] J. de Boer, F. Harmsze, and T. Tjin, Nonlinear finite W-symmetries and applications in elementary systems, Phys. Rep. 272, 139 (1996).

[69] J. M. Guilarte and M. S. Plyushchay, Nonlinear symmetries of perfectly invisible PT-regularized conformal and superconformal mechanics systems, J. High Energy Phys. 01 (2019) 194.

[70] M. S. Plyushchay, Nonlinear supersymmetry as a hidden symmetry, arXiv:1811.11942.

[71] U. Niederer, The maximal kinematical invariance group of the harmonic oscillator, Helv. Phys. Acta 46, 191 (1973).

[72] J. Gonera, Conformal mechanics, Ann. Phys. (Amsterdam) 335, 61 (2013).

[73] O. Steuernagel, Equivalence between free quantum particles and those in harmonic potentials and its application to instantaneous changes, Eur. Phys. J. Plus 129, 114 (2014).

[74] T. Okazaki, Implications of conformal symmetry in quantum mechanics, Phys. Rev. D 96, 066030 (2017).

[75] H. Airault, H. P. McKean, and J. Moser, Rational and elliptic solutions of the Korteweg-de Vries equation and a related many-body problem, Commun. Pure Appl. Math. 30, 95 (1977).

[76] M. Adler and J. Moser, On a class of polynomials connected with the Korteweg-deVries equation, Commun. Math. Phys. 61, 1 (1978).

[77] A. Gorsky and N. Nekrasov, Hamiltonian systems of Calogero type and two-dimensional Yang-Mills theory, Nucl. Phys. B414, 213 (1994). 